TRANSACTIONS OF THE

AMERICAN MATHEMATICAL SOCIETY

Volume 358, Number 3, Pages 1257-1283

S 0002-9947(05)03771-2

Article electronically published on May 26, 2005

\title{
GORENSTEIN PROJECTIVE DIMENSION FOR COMPLEXES
}

\author{
OANA VELICHE
}

\begin{abstract}
We define and study a notion of Gorenstein projective dimension for complexes of left modules over associative rings. For complexes of finite Gorenstein projective dimension we define and study a Tate cohomology theory. Tate cohomology groups have a natural transformation to classical Ext groups. In the case of module arguments, we show that these maps fit into a long exact sequence, where every third term is a relative cohomology group defined for left modules of finite Gorenstein projective dimension.
\end{abstract}

\section{INTRODUCTION}

We study generalized homological dimensions for complexes of modules. More precisely we define and study a notion of Gorenstein projective dimension for complexes of left modules over associative rings.

In the classical book of Cartan and Eilenberg [10] concepts of projective, injective and weak (flat) dimensions were defined for left modules over arbitrary rings. New dimensions have been defined since then. For finite modules over commutative noetherian rings Auslander and Bridger [1] introduced a Gorenstein dimension. The reason for the name is that a commutative local ring is Gorenstein if and only if every finite module has finite Gorenstein dimension. More recently, several dimensions have been defined over commutative noetherian local rings: complete intersection dimension by Avramov, Gasharov and Peeva [5], polynomial complete intersection dimension by Gerko [17, upper Gorenstein projective dimension by Veliche 24] and Cohen-Macaulay dimension by Gerko [17. These dimensions are related to the ring in the same way as the Gorenstein dimension is related to the Gorenstein rings. An overview of these dimensions can be found in [2].

For every left module $M$ over an associative ring $R$, Enochs and Jenda [13] defined a Gorenstein projective dimension denoted $\operatorname{Gpd}_{R} M$; they studied it when the ring is coherent or $n$-Gorenstein. For finite modules over commutative noetherian rings it coincides with the Auslander and Bridger's Gorenstein dimension. For left modules over arbitrary associative rings, Holm [20] proved that the new concept has the desired properties.

In a different direction, homological dimensions have been extended to complexes. Avramov and Foxby [3] defined projective, injective, and flat dimensions for arbitrary complexes of left modules over associative rings. Over commutative

Received by the editors October 8, 2003 and, in revised form, May 8, 2004.

2000 Mathematics Subject Classification. Primary 16E10, 18G25, 13D05; Secondary 13D25, $16 \mathrm{E} 30,16 \mathrm{E} 45$. 
local rings, Yassemi 23] and Christensen 12] introduced a Gorenstein projective dimension for complexes with bounded below homology. Complete intersection dimension was extended to homologically finite complexes by Sather-Wagstaff 22.

The main purpose of this paper is to introduce and study a concept of Gorenstein projective dimension $\operatorname{Gpd}_{R} M$ associated to every (not necessarily bounded) complex $\boldsymbol{M}$ of left modules over an arbitrary associative ring $R$. It is obtained by blending the approach of Enochs and Jenda to the Gorenstein projective dimension of modules with that of Avramov and Martsinkovsky [6] to the Gorenstein dimension of finite modules over noetherian rings. We show that the previously defined notions, when they can be applied, agree with ours, and that most properties of modules of finite Gorenstein projective dimension are preserved for complexes. Next we describe our construction and some results in more detail.

A complex $\boldsymbol{T}$ is called totally acyclic if its modules are projective, it is exact, and the complex $\operatorname{Hom}_{R}(\boldsymbol{T}, Q)$ is exact for every projective module $Q$. We say that a complex $\boldsymbol{M}$ has $\operatorname{Gpd}_{R} \boldsymbol{M} \leq g$ if and only if there exist morphisms of complexes

$$
\boldsymbol{T} \stackrel{\tau}{\rightarrow} \boldsymbol{P} \stackrel{\pi}{\rightarrow} M,
$$

where $\boldsymbol{T}$ is totally acyclic, $\tau_{i}$ is bijective for all $i \geq g$ (with the grading convention from 1.1), and $\boldsymbol{P} \stackrel{\pi}{\rightarrow} \boldsymbol{M}$ is a semiprojective resolution (in the sense of [4); such a diagram is called a complete resolution of $\boldsymbol{M}$. In 3.4 we prove that the Gorenstein projective dimension of $\boldsymbol{M}$ can be computed from any semiprojective resolution $\boldsymbol{P} \rightarrow \boldsymbol{M}$. In 3.7 we relate it to projective dimension by an inequality $\operatorname{Gpd}_{R} \boldsymbol{M} \leq$ $\operatorname{pd}_{R} \boldsymbol{M}$, where equality holds when the right-hand side is finite. When $\operatorname{Gpd}_{R} \boldsymbol{M}$ is finite, we prove in 3.8 that it is equal to the least integer $n$ for which $\operatorname{Ext}_{R}^{i}(\boldsymbol{M}, Q)=$ 0 for all $i \geq n$ and all projective modules $Q$.

Tate cohomology for finite modules of finite Gorenstein dimension over noetherian rings was treated, most recently, by Avramov and Martsinkovsky in 6]. In Section 4 we extend their construction to the case of complexes and show that most of its properties for modules extend well to the framework of complexes. For each complex $\boldsymbol{M}$ of finite Gorenstein projective dimension and every complex $\boldsymbol{N}$, the Tate cohomology $\operatorname{Ext}_{R}(\boldsymbol{M}, \boldsymbol{N})$ is the cohomology of the complex $\operatorname{Hom}_{R}(\boldsymbol{T}, \boldsymbol{N})$, where $\boldsymbol{T} \rightarrow \boldsymbol{P} \rightarrow \boldsymbol{M}$ is a complete resolution. It is easily seen to be a covariant functor of the second argument. In 4.8 we prove that it is a contravariant functor in the first argument; this is rather more delicate. As in the case of modules, Tate cohomology is rigid: In 4.5 we prove that $\operatorname{Ext}_{R}^{i}(\boldsymbol{M},-)=0$ for a single $i \in \mathbb{Z}$ is equivalent to $\operatorname{pd}_{R} \boldsymbol{M}<\infty$. If $\boldsymbol{M}$ is bounded below, then we also prove that $\operatorname{pd}_{R} \boldsymbol{M}<\infty$ if and only if $\operatorname{Ext}_{R}^{i}(-, \boldsymbol{M})=$ for some (any) $i \in \mathbb{Z}$, if and only if $\mathrm{Ext}_{R}^{0}(\boldsymbol{M}, \boldsymbol{M})=0$.

In Section 5 we show that when the complex $\boldsymbol{M}$ has bounded homology, $\operatorname{Gpd}_{R} \boldsymbol{M}$ can be expressed in terms of finite and of special Gorenstein projective resolutions; see 5.3. In particular, we show in 5.4 that our definition of Gorenstein projective dimension agrees with the ones of Gorenstein (projective) dimension of Christensen and Yassemi when they can be applied.

In the last section, we show the functorial properties of relative cohomology for left modules over associative rings and show in 6.6 that for any module of finite Gorenstein projective dimension there exists a long exact sequence relating the natural Ext, Tate cohomology and relative cohomology. 


\section{Complexes}

In this section we recall basic definitions and properties of complexes used in this paper. For more details the reader can consult [4] or [21].

1.1. Complexes. Let $R$ be an associative ring, $\mathcal{M}=\mathcal{M}(R)$ the category of left $R$-modules and $\mathcal{C}=\mathcal{C}(R)$ the category of complexes of left $R$-modules.

To every complex

$$
\boldsymbol{M}=\cdots \rightarrow M_{n} \stackrel{\partial_{n}^{M}}{\longrightarrow} M_{n-1} \rightarrow \cdots
$$

in $\mathcal{C}$ we associate the numbers

$$
\sup \boldsymbol{M}=\sup \left\{i \mid M_{i} \neq 0\right\} \quad \text { and } \quad \inf \boldsymbol{M}=\inf \left\{i \mid M_{i} \neq 0\right\} .
$$

The complex $\boldsymbol{M}$ is called bounded above when sup $\boldsymbol{M}<\infty$, bounded below when $\inf \boldsymbol{M}>-\infty$ and bounded when it is bounded below and above.

The $n^{\text {th }}$ homology module of $\boldsymbol{M}$ is the module $\mathrm{H}_{n}(\boldsymbol{M})=\operatorname{Ker}\left(\partial_{n}^{\boldsymbol{M}}\right) / \operatorname{Im}\left(\partial_{n+1}^{\boldsymbol{M}}\right)$; we set $\mathrm{H}^{n}(\boldsymbol{M})=\mathrm{H}_{-n}(\boldsymbol{M})$. We also set $\mathrm{C}_{n}(\boldsymbol{M})=\operatorname{Coker}\left(\partial_{n+1}^{\boldsymbol{M}}\right)$. The $i^{\text {th }}$ shift of $\boldsymbol{M}$ is a complex $\Sigma^{i} \boldsymbol{M}$ with $n^{\text {th }}$ component $M_{n-i}$ and differential $\partial_{n}^{\Sigma^{i} \boldsymbol{M}}=$ $(-1)^{i} \partial_{n-i}^{M}$; we write $\Sigma \boldsymbol{M}$ instead of $\Sigma^{1} \boldsymbol{M}$.

In the following discussion, $\boldsymbol{M}$ and $\boldsymbol{N}$ denote complexes of left $R$-modules.

1.1.1. A homomorphism $\varphi: M \rightarrow N$ of degree $n$ is a family $\left(\varphi_{i}\right)_{i \in \mathbb{Z}}$ of homomorphisms of $R$-modules $\varphi_{i}: M_{i} \rightarrow N_{i+n}$. All such homomorphisms form an abelian group, denoted $\operatorname{Hom}_{R}(\boldsymbol{M}, \boldsymbol{N})_{n}$; it is clearly isomorphic to $\prod_{i \in \mathbb{Z}} \operatorname{Hom}_{R}\left(M_{i}, N_{i+n}\right)$. We let $\operatorname{Hom}_{R}(\boldsymbol{M}, \boldsymbol{N})$ denote the complex of $\mathbb{Z}$-modules with $n^{\text {th }}$ component $\operatorname{Hom}_{R}(\boldsymbol{M}, \boldsymbol{N})_{n}$ and differential

$$
\partial(\varphi)=\partial^{N} \varphi-(-1)^{|\varphi|} \varphi \partial^{M} .
$$

A homomorphism $\varphi \in \operatorname{Hom}_{R}(\boldsymbol{M}, \boldsymbol{N})_{n}$ is called a chain map if $\partial(\varphi)=0$, i.e., if

$$
\partial_{i+n}^{N} \varphi_{i}=(-1)^{|\varphi|} \varphi_{i-1} \partial_{i}^{M} \quad \text { for all } \quad i \in \mathbb{Z} \text {. }
$$

A chain map of degree 0 is called a morphism. Homomorphisms $\varphi$ and $\varphi^{\prime}$ in $\operatorname{Hom}_{R}(\boldsymbol{M}, \boldsymbol{N})_{n}$ are called homotopic, denoted $\varphi \sim \varphi^{\prime}$, if there exists a degree $n+1$ homomorphism $\varkappa$, called a homotopy, such that $\partial(\varkappa)=\varphi-\varphi^{\prime}$. A homotopy equivalence is a morphism $\varphi: M \rightarrow \boldsymbol{N}$ for which there exists a morphism $\psi: \boldsymbol{N} \rightarrow$ $\boldsymbol{M}$ such that $\varphi \psi \sim \mathrm{id}^{N}$ and $\psi \varphi \sim \operatorname{id}^{M}$.

1.1.2 ([4, (6.2.7)]). Every morphism $\varphi: M \rightarrow \boldsymbol{N}$ has a factorization

$$
\boldsymbol{M} \stackrel{\mu}{\longrightarrow} M^{\prime} \stackrel{\varphi^{\prime}}{\longrightarrow} N
$$

where $\boldsymbol{M}^{\prime}=\boldsymbol{M} \oplus \operatorname{Cone}\left(\mathrm{id}^{\boldsymbol{N}}\right), \mu$ is a homotopy equivalence and $\varphi^{\prime}$ is a surjective morphism.

1.1.3. The complex $\boldsymbol{M}$ is called contractible if it is homotopy equivalent to $\mathbf{0}$. This is the case if and only if $\operatorname{Hom}_{R}(\boldsymbol{M}, \boldsymbol{M})$ is exact, if and only if $\boldsymbol{M} \cong \mathrm{Cone}\left(\mathrm{id}^{\boldsymbol{Z}}\right)$ where $\boldsymbol{Z}$ is a complex with trivial differential; see [4, (6.2.8)].

1.1.4. A quasi-isomorphism $\varphi: M \rightarrow \boldsymbol{N}$ is a morphism, such that the induced map $\mathrm{H}_{n}(\varphi): \mathrm{H}_{n}(\boldsymbol{M}) \rightarrow \mathrm{H}_{n}(\boldsymbol{N})$ is an isomorphism for all $n \in \mathbb{Z}$. The complexes $\boldsymbol{M}$ and $\boldsymbol{N}$ are called quasi-isomorphic, and denoted by $\boldsymbol{M} \simeq \boldsymbol{N}$, if they can be linked by a sequence of quasi-isomorphisms with arrows in alternating directions. 
1.1.5 ([ㅁ, (1.1.1)]). Let $\beta: N \rightarrow N^{\prime}$ be a morphism of complexes such that

$$
\operatorname{Hom}_{R}(\boldsymbol{M}, \beta): \operatorname{Hom}_{R}(\boldsymbol{M}, \boldsymbol{N}) \rightarrow \operatorname{Hom}_{R}\left(\boldsymbol{M}, \boldsymbol{N}^{\prime}\right)
$$

is a quasi-isomorphism. For each morphism $\gamma: \boldsymbol{M} \rightarrow \boldsymbol{N}^{\prime}$ there is a morphism $\alpha: \boldsymbol{M} \rightarrow \boldsymbol{N}$ with $\gamma \sim \beta \alpha$ (even $\gamma=\beta \alpha$ if $\operatorname{Hom}_{R}(\boldsymbol{M}, \beta)$ is surjective). If $\gamma^{\prime}: \boldsymbol{M} \rightarrow$ $\boldsymbol{N}^{\prime}$ and $\alpha^{\prime}: \boldsymbol{M} \rightarrow \boldsymbol{N}$ satisfy $\gamma^{\prime} \sim \gamma$ and $\gamma^{\prime} \sim \beta \alpha^{\prime}$, then $\alpha^{\prime} \sim \alpha$.

From [7, (§5, no. 2, Proposition 2)] and [4, (4.2.3), (8.2.4)] we obtain

1.1.6. If $\boldsymbol{P}$ is a complex of projectives, then $\operatorname{Hom}_{R}(\boldsymbol{P},-)$ preserves quasiisomorphisms between bounded below complexes.

1.2. Connecting homomorphisms. Let $M_{\bullet}=0 \rightarrow M \stackrel{\mu}{\rightarrow} M^{\prime} \stackrel{\mu^{\prime}}{\rightarrow} M^{\prime \prime} \rightarrow 0$ be an exact sequence of complexes which splits as a sequence of graded modules. There exist homomorphisms $\nu^{\prime \prime}: \boldsymbol{M}^{\prime \prime} \rightarrow \boldsymbol{M}^{\prime}$ and $\nu^{\prime}: \boldsymbol{M}^{\prime} \rightarrow \boldsymbol{M}$ such that

$$
\nu^{\prime} \nu^{\prime \prime}=0, \quad \mu^{\prime} \nu^{\prime \prime}=\mathrm{id}^{M^{\prime \prime}}, \quad \nu^{\prime} \mu=\mathrm{id}^{M} \quad \text { and } \quad \nu^{\prime \prime} \mu^{\prime}+\mu \nu^{\prime}=\mathrm{id}^{M^{\prime}} .
$$

Set $\theta^{M^{\prime \prime}}=\nu^{\prime} \partial^{M^{\prime}} \nu^{\prime \prime}$.

1.2.1 (21, (4.8), p. 19]). The map $\theta^{\boldsymbol{M}^{\prime \prime}}: \boldsymbol{M}^{\prime \prime} \rightarrow \Sigma \boldsymbol{M}$ is a morphism of complexes and $\partial=\mathrm{H}\left(\theta^{M^{\prime \prime}}\right)$, where $\partial$ is the connecting homomorphism of the cohomology long exact sequence of $\boldsymbol{M}_{\text {. }}$.

1.2.2 ([21, (4.9), p. 20]). For any complex $\boldsymbol{N}$ the connecting homomorphism of the long exact homology sequence

$$
0 \rightarrow \operatorname{Hom}_{R}\left(\boldsymbol{M}^{\prime \prime}, \boldsymbol{N}\right) \rightarrow \operatorname{Hom}_{R}\left(\boldsymbol{M}^{\prime}, \boldsymbol{N}\right) \rightarrow \operatorname{Hom}_{R}(\boldsymbol{M}, \boldsymbol{N}) \rightarrow 0
$$

is given by

$$
\check{\partial}_{R}^{n-1}\left(M_{\bullet}, \boldsymbol{N}\right)=(-1)^{n} \mathrm{H}^{n}\left(\operatorname{Hom}_{R}\left(\theta^{\boldsymbol{M}^{\prime \prime}}, \boldsymbol{N}\right)\right) \text { for all } n \in \mathbb{Z} .
$$

1.2.3. Let $\lambda: M \rightarrow N$ be a morphism of complexes. From the exact sequence

$$
0 \rightarrow \boldsymbol{N} \rightarrow \text { Cone }(\lambda) \rightarrow \Sigma \boldsymbol{M} \rightarrow 0
$$

we get a short exact sequence which splits as a sequence of graded modules

$$
0 \rightarrow \Sigma^{-1} \boldsymbol{N} \stackrel{\mu}{\rightarrow} \Sigma^{-1} \text { Cone }(\lambda) \stackrel{\mu^{\prime}}{\longrightarrow} \boldsymbol{M} \rightarrow 0 .
$$

Let $\nu^{\prime \prime}: M \rightarrow \Sigma^{-1}$ Cone $(\lambda)$ be the canonical injection and let $\nu^{\prime}: \Sigma^{-1}$ Cone $(\lambda) \rightarrow$ $\Sigma^{-1} N$ be the canonical surjection. Then, the map $\theta^{M}$ defined in 1.2 is given by

$$
\theta^{M}=\lambda \text {. }
$$

1.3. Semiprojective resolutions. Let $\boldsymbol{P}$ be a complex of $R$-modules.

Following [4], we say that $\boldsymbol{P}$ is semiprojective if $\operatorname{Hom}_{R}(\boldsymbol{P},-)$ preserves surjective quasi-isomorphisms.

1.3.1 (4 4 (8.5.1), (4.2.4.1)]). The following conditions are equivalent:

(i) $\boldsymbol{P}$ is semiprojective.

(ii) Each $P_{i}$ is projective and $\operatorname{Hom}_{R}(\boldsymbol{P},-)$ preserves quasi-isomorphisms.

(iii) Each $P_{i}$ is projective and $\mathrm{H}\left(\operatorname{Hom}_{R}(\boldsymbol{P}, \boldsymbol{N})\right)=0$ for every complex $\boldsymbol{N}$ with $\mathrm{H}(\boldsymbol{N})=0$.

1.3.2 ([3, (1.4.P)]). If $\boldsymbol{P}$ is a semiprojective complex and $\boldsymbol{P} \simeq \boldsymbol{M}$, then there exists a quasi-isomorphism $\boldsymbol{P} \rightarrow \boldsymbol{M}$. 
From 1.1.3 and 1.3.1 we get the following.

1.3.3. If $\boldsymbol{P}$ is a semiprojective complex with $\mathrm{H}(\boldsymbol{P})=0$, then $\boldsymbol{P}$ is contractible.

A semiprojective resolution of $\boldsymbol{M}$ is a quasi-isomorphism of complexes $\pi: \boldsymbol{P} \rightarrow$ $\boldsymbol{M}$, with $\boldsymbol{P}$ semiprojective; when $\pi$ is surjective, the resolution is called strict.

1.3.4 (4, (8.3.2), (11.2.4), (11.2.3)]). Every complex $\boldsymbol{M}$ has a strict semiprojective resolution $\boldsymbol{P} \rightarrow \boldsymbol{M}$. If $\mathrm{H}(\boldsymbol{M})$ is bounded below, then $\boldsymbol{P}$ can be chosen so that $\inf \boldsymbol{P}=\inf \mathrm{H}(\boldsymbol{M})$. If, in addition, $R$ is left noetherian and $\mathrm{H}_{i}(\boldsymbol{M})$ is finitely generated for each $i \in \mathbb{Z}$, then $\boldsymbol{P}$ can be chosen with each $P_{i}$ finitely generated.

1.3.5 (4, (8.3.1)]). If $M$ is a left module, then any classical projective resolution $\boldsymbol{P} \rightarrow M$ is a strict semiprojective resolution.

The next two results were communicated to the author by Luchezar Avramov. They are special cases of theorems that will eventually be included in [4].

Proposition 1.3.6 (Shanuel's Lemma for Complexes). If $\boldsymbol{P} \rightarrow \boldsymbol{M}$ and $\boldsymbol{P}^{\prime} \rightarrow \boldsymbol{M}$ are semiprojective resolutions, then for every $n \in \mathbb{Z}$ there exist projective modules $Q_{n}$ and $Q_{n}^{\prime}$, such that

$$
\mathrm{C}_{n}(\boldsymbol{P}) \oplus Q_{n}^{\prime} \cong \mathrm{C}_{n}\left(\boldsymbol{P}^{\prime}\right) \oplus Q_{n} .
$$

Proof. By 1.3.2, there exists a comparison of semiprojective resolutions $\boldsymbol{P}^{\prime} \rightarrow \boldsymbol{P}$. Using 1.1.2, one can factor it as a composition of quasi-isomorphisms

$$
\boldsymbol{P}^{\prime} \rightarrow \boldsymbol{P}^{\prime \prime} \stackrel{\varphi}{\rightarrow} \boldsymbol{P}
$$

where $\boldsymbol{P}^{\prime \prime}=\boldsymbol{P}^{\prime} \oplus$ Cone $\left(\mathrm{id}^{\boldsymbol{P}}\right)$ and $\varphi$ is surjective. For each $n \in \mathbb{Z}$ we now have

$$
\mathrm{C}_{n}\left(\boldsymbol{P}^{\prime \prime}\right)=\mathrm{C}_{n}\left(\boldsymbol{P}^{\prime}\right) \oplus \mathrm{C}_{n}\left(\text { Cone }\left(\text { id }^{\boldsymbol{P}}\right)\right) .
$$

The complex Cone $\left(\mathrm{id}^{\boldsymbol{P}}\right)$ is contractible, so the module $Q_{n}=\mathrm{C}_{n}\left(\mathrm{Cone}\left(\mathrm{id}^{\boldsymbol{P}}\right)\right)$ is projective. On the other hand, one has an exact sequence of complexes

$$
0 \rightarrow \boldsymbol{Q}^{\prime} \rightarrow \boldsymbol{P}^{\prime \prime} \stackrel{\varphi}{\rightarrow} \boldsymbol{P} \rightarrow 0 .
$$

Since $\varphi$ is a quasi-isomorphism, we conclude that $\mathrm{H}\left(\boldsymbol{Q}^{\prime}\right)=0$. Because, in addition, $\boldsymbol{P}$ is semiprojective, the sequence splits yielding an isomorphism of complexes

$$
\boldsymbol{P}^{\prime \prime} \cong \boldsymbol{P} \oplus \boldsymbol{Q}^{\prime}
$$

It shows that $\boldsymbol{Q}^{\prime}$ is semiprojective and produces an isomorphism of modules

$$
\mathrm{C}_{n}\left(\boldsymbol{P}^{\prime \prime}\right) \cong \mathrm{C}_{n}(\boldsymbol{P}) \oplus \mathrm{C}_{n}\left(\boldsymbol{Q}^{\prime}\right) .
$$

By 1.3.3, $\boldsymbol{Q}^{\prime}$ is contractible, so the module $Q_{n}^{\prime}=\mathrm{C}_{n}\left(\boldsymbol{Q}^{\prime}\right)$ is projective.

1.3.7. We define the $n^{\text {th }}$ syzygy of a complex $\boldsymbol{M}$ to be $\Omega_{n}(\boldsymbol{M})=\mathrm{C}_{n}(\boldsymbol{P})$, where $\boldsymbol{P} \rightarrow \boldsymbol{M}$ is a semiprojective resolution. In view of Proposition 1.3.6, $\Omega_{n} \boldsymbol{M}$ is defined uniquely up to a projective direct summand.

Proposition 1.3.8 (Horseshoe Lemma). For every exact sequence of complexes

$$
0 \rightarrow M \stackrel{\mu}{\longrightarrow} M^{\prime} \stackrel{\mu^{\prime}}{\longrightarrow} M^{\prime \prime} \rightarrow 0
$$


there exists a commutative diagram with exact rows

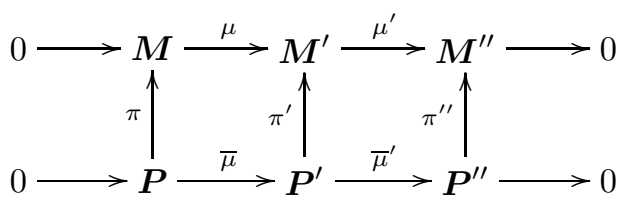

in which the columns are strict semiprojective resolutions.

Proof. Using 1.3.4, choose strict semiprojective resolutions $\pi: P \rightarrow M$ and $\varkappa: Q \rightarrow$ $\boldsymbol{M}^{\prime}$. Set $\boldsymbol{P}^{\prime}=$ Cone $\left(\mathrm{id}^{\boldsymbol{P}}\right) \oplus \boldsymbol{Q}$, and note that this complex is semiprojective. The map $\pi^{\prime}: \boldsymbol{P}^{\prime} \rightarrow \boldsymbol{M}^{\prime}$ defined by $\pi^{\prime}\left(p^{\prime}, p, q\right)=\varkappa(q)$ is a composition of the canonical epimorphism Cone(id $\left.\boldsymbol{P}^{\boldsymbol{P}}\right) \oplus \boldsymbol{Q} \rightarrow \boldsymbol{Q}$, whose kernel Cone(id $\left.{ }^{\boldsymbol{P}}\right)$ is contractible, with the quasi-isomorphism $\varkappa$. It follows that $\pi^{\prime}$ is a quasi-isomorphism, hence $\pi^{\prime}$ is a strict semiprojective resolution.

Choose, by 1.1.5 a morphism $\alpha: \boldsymbol{P} \rightarrow \boldsymbol{Q}$ such that $\varkappa \alpha=\mu \pi$ and define a map $\bar{\mu}: \boldsymbol{P} \rightarrow \boldsymbol{P}^{\prime}$ by $\bar{\mu}(p)=(0, p, \alpha(p))$. It is easy to check that this is an injective morphism. Set $\boldsymbol{P}^{\prime \prime}=$ Coker $\bar{\mu}$, and form a diagram (図), where $\bar{\mu}^{\prime}$ denotes the canonical surjection and $\pi^{\prime \prime}: \boldsymbol{P}^{\prime \prime} \rightarrow \boldsymbol{M}^{\prime \prime}$ is the unique morphism induced by $\pi^{\prime}$ satisfying $\pi^{\prime \prime} \bar{\mu}^{\prime}=\mu^{\prime} \pi^{\prime}$. The resulting diagram is commutative with exact rows.

It remains to prove that $\pi^{\prime \prime}: \boldsymbol{P}^{\prime \prime} \rightarrow \boldsymbol{M}^{\prime \prime}$ is a strict semiprojective resolution. This morphism is surjective, because $\mu^{\prime}$ and $\pi^{\prime}$ are. It is a quasi-isomorphism by the Five-Lemma, because $\pi$ and $\pi^{\prime}$ are. For each $n$ define a map $\beta_{n}: P_{n}^{\prime} \longrightarrow P_{n-1} \oplus Q_{n}$ by $\beta\left(p^{\prime}, p, q\right)=\left(p^{\prime}, q-\alpha(p)\right)$. These maps are clearly $R$-linear, surjective, with $\operatorname{Ker} \beta_{n}=\operatorname{Im} \bar{\mu}_{n}$, so they produce isomorphisms $P_{n}^{\prime \prime} \cong P_{n-1} \oplus Q_{n}$. Thus, every $R$-module $P_{n}^{\prime \prime}$ is projective. We now know that the lower row of the diagram splits as a sequence of graded $R$-modules, so each complex $N$ induces an exact sequence

$$
0 \longrightarrow \operatorname{Hom}_{R}\left(\boldsymbol{P}^{\prime \prime}, \boldsymbol{N}\right) \longrightarrow \operatorname{Hom}_{R}\left(\boldsymbol{P}^{\prime}, \boldsymbol{N}\right) \longrightarrow \operatorname{Hom}_{R}(\boldsymbol{P}, \boldsymbol{N}) \longrightarrow 0 .
$$

As $\boldsymbol{P}^{\prime}$ and $\boldsymbol{P}$ are semiprojective, $\mathrm{H}(\boldsymbol{N})=0$ implies $\mathrm{H}\left(\operatorname{Hom}_{R}\left(\boldsymbol{P}^{\prime}, \boldsymbol{N}\right)\right)=0$ and $\mathrm{H}\left(\operatorname{Hom}_{R}(\boldsymbol{P}, \boldsymbol{N})\right)=0$ by 1.3.1. From the homology exact sequence one now gets $\mathrm{H}\left(\operatorname{Hom}_{R}\left(\boldsymbol{P}^{\prime \prime}, \boldsymbol{N}\right)\right)=0$, so $\boldsymbol{P}^{\prime \prime}$ is semiprojective by 1.3.1. Summing up the preceding discussion, we see that $\pi^{\prime \prime}: \boldsymbol{P}^{\prime \prime} \rightarrow \boldsymbol{M}^{\prime \prime}$ is a strict semiprojective resolution.

1.4. Projective dimension. In 3 the projective dimension of $\boldsymbol{M}$ is defined to be the infimum of those $p \in \mathbb{Z}$ for which there exists a semiprojective resolution $\boldsymbol{P} \rightarrow \boldsymbol{M}$ with $P_{i}=0$ for all $i>p$; when no such $p$ exits, $\boldsymbol{M}$ is said to have infinite projective dimension. The projective dimension of $\boldsymbol{M}$ is denoted $\operatorname{pd}_{R} \boldsymbol{M}$. When $M$ is a module, this number is equal to the classical invariant.

1.4.1 ([3, (2.4.P)]). For each integer $p$ the following conditions are equivalent:

(i) $\operatorname{pd}_{R} \boldsymbol{M} \leq p$.

(ii) $\sup \mathrm{H}(\boldsymbol{M}) \leq p$ and there exists a semiprojective resolution $\boldsymbol{P} \rightarrow \boldsymbol{M}$ such that the module $\mathrm{C}_{p}(\boldsymbol{P})$ is projective.

(ii') $\sup \mathrm{H}(\boldsymbol{M}) \leq p$ and for every semiprojective resolution $\boldsymbol{P}^{\prime} \rightarrow \boldsymbol{M}$ the module $\mathrm{C}_{p}\left(\boldsymbol{P}^{\prime}\right)$ is projective.

(iii) $\sup H(\boldsymbol{M}) \leq p$ and $\Omega_{p}(\boldsymbol{M})$ is projective. 
1.4.2 ([3, (2.4.P)]). There are equalities

$$
\begin{aligned}
\operatorname{pd}_{R} \boldsymbol{M} & =\sup \left\{\begin{array}{l|l}
n \in \mathbb{Z} & \begin{array}{c}
\operatorname{Ext}_{R}^{n}(\boldsymbol{M}, N) \neq 0 \\
\text { for some module } N
\end{array}
\end{array}\right\} \\
& =\sup \left\{\begin{array}{l|c}
n \in \mathbb{Z} & \begin{array}{c}
\operatorname{Ext}_{R}^{n-\inf \mathrm{H}(\boldsymbol{N})}(\boldsymbol{M}, \boldsymbol{N}) \neq 0 \\
\text { for some complex } \boldsymbol{N} \text { with } \inf \mathrm{H}(\boldsymbol{N})>-\infty
\end{array}
\end{array}\right\} .
\end{aligned}
$$

Thus, the projective dimension of complexes can be computed in terms of vanishing of appropriate Ext groups, as is the case for modules.

An immediate consequence of Proposition 1.3.8 and 1.4 .2 is the following.

1.4.3. Let $0 \rightarrow \boldsymbol{M} \rightarrow \boldsymbol{M}^{\prime} \rightarrow \boldsymbol{M}^{\prime \prime} \rightarrow 0$ be an exact sequence of complexes.

(1) If two complexes have finite projective dimension, then so does the third.

(2) There is an inequality

$$
\operatorname{pd}_{R} \boldsymbol{M}^{\prime} \leq \max \left(\operatorname{pd}_{R} \boldsymbol{M}, \operatorname{pd}_{R} \boldsymbol{M}^{\prime \prime}\right)
$$

and equality holds, except possibly when $\operatorname{pd}_{R} \boldsymbol{M}^{\prime \prime}=\operatorname{pd}_{R} \boldsymbol{M}+1$.

\section{Totally ACYCLIC COMPLEXES}

In this section we first review the notions of totally acyclic complex and complete resolution, which are at the basis of the definition of Gorenstein projective dimension. Second, we collect properties of modules of finite Gorenstein projective dimension of modules that will be used to define and study Gorenstein projective dimension and Tate cohomology for complexes. Finally, we compare the notion of Gorenstein projective dimension with the earlier notion of Gorenstein dimension, which is defined only for finite modules over noetherian rings.

2.1. Total acyclicity. The following notion is fundamental to our investigation.

2.1.1. A complex of $R$-modules $\boldsymbol{T}$ is said to be totally acyclic if the following conditions are satisfied:

(1) $T_{n}$ is projective for every $n \in \mathbb{Z}$.

(2) $\boldsymbol{T}$ is exact.

(3) $\operatorname{Hom}_{R}(\boldsymbol{T}, Q)$ is exact for every projective $R$-module $Q$.

This meaning of "totally acyclic" is wider than the one in [6], where it refers to an exact complex $\boldsymbol{T}$ of finite projective modules such that $\operatorname{Hom}_{R}(\boldsymbol{T}, R)$ is exact; when $R$ is noetherian, the new notion subsumes the earlier one, by [6, (2.4)].

We shall use the following result of Cornick and Kropholler.

2.1.2 ([11, (2.4)]). Let $\boldsymbol{T}$ be a totally acyclic complex. If $\boldsymbol{Q}$ is a complex of projectives and $n$ is an integer, then any chain map $\underline{\phi}: \boldsymbol{T}_{\geq n} \rightarrow \boldsymbol{Q}_{\geq n}$ can be extended to a chain map $\phi: \boldsymbol{T} \rightarrow \boldsymbol{Q}$ such that $\phi_{\geq n}=\underline{\phi}$. Every chain map $\phi$ with this property is defined as unique up to homotopy.

As a consequence we obtain the following.

2.1.3. Let $\boldsymbol{T}$ be a totally acyclic complex. If $\boldsymbol{Q}$ is a bounded above complex of projectives, then

$$
\mathrm{H}\left(\operatorname{Hom}_{R}(\boldsymbol{T}, \boldsymbol{Q})\right)=0 .
$$


Indeed, set $\sup \boldsymbol{Q}=s$. If $\alpha: \boldsymbol{T} \rightarrow \boldsymbol{Q}$ is a chain map of degree $n$, then $\alpha_{\geq s-n+1}$ is the zero map. The preceding result shows that $\alpha$ is homotopic to 0 , that is, $\mathrm{H}_{n}\left(\operatorname{Hom}_{R}(\boldsymbol{T}, \boldsymbol{Q})\right)=0$.

2.2. Complete resolutions. A notion of complete resolution was initially introduced, for finite modules over finite groups, by Tate [10, Ch. XII]. Cornick and Kropholler used it in 11, in a modified form; more precisely, they added condition (3) in the definition of a totally acyclic complex 2.1.1. Avramov and Martsinkovsky [6] incorporated a comparison morphism to a projective resolution, in the notion of complete resolution for finite modules over noetherian rings. Using this last perspective, we extend the notion of complete resolutions to complexes.

Let $\boldsymbol{M}$ be a complex of $R$-modules.

2.2.1. A complete resolution of $\boldsymbol{M}$ is a diagram of morphisms of complexes $\boldsymbol{T} \stackrel{\tau}{\rightarrow}$ $\boldsymbol{P} \stackrel{\pi}{\longrightarrow} \boldsymbol{M}$ where $\pi: \boldsymbol{P} \rightarrow \boldsymbol{M}$ is a semiprojective resolution, $\boldsymbol{T}$ is a totally acyclic complex and $\tau_{i}$ is bijective for all $i \gg 0$. A complete resolution is said to be surjective if $\tau_{i}$ is surjective for all $i \in \mathbb{Z}$.

The next result shows that from any complete resolution one can get a surjective complete resolution. The proof given in [6, (3.7)] extends easily and is omitted.

2.2.2. Let $\boldsymbol{T} \stackrel{\tau}{\rightarrow} \boldsymbol{P} \stackrel{\pi}{\longrightarrow} \boldsymbol{M}$ be a complete resolution. If $g$ is an integer such that $\tau_{i}$ is bijective for all $i \geq g$, then there exists a complete resolution $\boldsymbol{T}^{\prime} \stackrel{\tau^{\prime}}{\rightarrow} \boldsymbol{P} \stackrel{\pi}{\rightarrow} \boldsymbol{M}$ where $\tau^{\prime}$ is a surjective morphism, such that $\tau_{i}^{\prime}$ is bijective for all $i \geq g$, and a homotopy equivalence $\alpha: \boldsymbol{T} \rightarrow \boldsymbol{T}^{\prime}$ such that $\tau=\tau^{\prime} \alpha$ and $\alpha_{i}=\operatorname{id}^{T_{i}}$ for all $i \geq g$.

2.2.3. Let $\boldsymbol{T} \stackrel{\tau}{\rightarrow} \boldsymbol{P} \stackrel{\pi}{\longrightarrow} \boldsymbol{M}$ be a surjective complete resolution and set $\boldsymbol{L}=\operatorname{Ker} \tau$. The short exact sequence of complexes

$$
0 \rightarrow \boldsymbol{L} \stackrel{\varkappa}{\longrightarrow} \boldsymbol{T} \stackrel{\tau}{\rightarrow} \boldsymbol{P} \rightarrow 0
$$

splits as a sequence of graded modules since $\boldsymbol{P}$ is a complex of projectives. Let $\lambda: \boldsymbol{P} \rightarrow \Sigma \boldsymbol{L}$ be the morphism $\theta^{\boldsymbol{P}}$ constructed in 1.2. By 1.2.1, we have $\mathrm{H}(\lambda)=\check{\partial}$, where $\partial$ is the connecting homomorphism of the homology exact sequence associated to the short exact sequence above. Since $\boldsymbol{T}$ is exact, $\lambda$ is a quasi-isomorphism.

When $\boldsymbol{M}$ and $\boldsymbol{M}^{\prime}$ are finite modules over noetherian rings, the following result is contained in the statement and the proof of [6, (5.3)]. The arguments carry over verbatim, so they are omitted.

Proposition 2.2.4. Let $\boldsymbol{T} \stackrel{\tau}{\rightarrow} \boldsymbol{P} \stackrel{\pi}{\rightarrow} \boldsymbol{M}$ and $\boldsymbol{T}^{\prime} \stackrel{\tau^{\prime}}{\longrightarrow} \boldsymbol{P}^{\prime} \stackrel{\pi^{\prime}}{\longrightarrow} \boldsymbol{M}^{\prime}$ be complete resolutions. For each morphism of complexes $\mu: \boldsymbol{M} \rightarrow \boldsymbol{M}^{\prime}$ there exists a unique up to homotopy morphism $\bar{\mu}$, making the right-hand square of the diagram

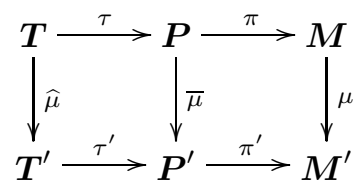

commute up to homotopy, and for each choice of $\bar{\mu}$ there exists a unique up to homotopy morphism $\widehat{\mu}$, making the left-hand square commute up to homotopy. Moreover, if $\pi^{\prime}$ and $\tau^{\prime}$ are surjective, then $\bar{\mu}$ and respectively $\widehat{\mu}$ can be chosen such that the right-hand square and respectively the left-hand side square commute.

If $\mu=\mathrm{id}^{M}$, then $\bar{\mu}$ and $\widehat{\mu}$ are homotopy equivalences. 
2.3. Gorenstein projective modules. A notion of Gorenstein projective dimension, for left modules over associative rings, is due to Enochs and Jenda [13. It extends the notion of Gorenstein dimension, introduced by Auslander and Bridger 1], for finite modules over commutative noetherian rings. Enochs and Jenda study it for left or right modules over coherent or $n$-Gorenstein rings and Holm 20] extends their results to arbitrary modules over associative rings. In this subsection we recall some definitions and give some basic results that are needed in this paper.

2.3.1. An $R$-module $G$ is Gorenstein projective if there exists a totally acyclic complex $\boldsymbol{T}$, as defined in 2.1.1, with $\mathrm{C}_{0}(\boldsymbol{T})=G$.

2.3.2 ([20, (2.20)]). If $G$ is Gorenstein projective and $Q$ is projective, then

$$
\operatorname{Ext}_{R}^{i}(G, Q)=0 \quad \text { for all } \quad i \geq 1 .
$$

Indeed, by definition there exists a totally acyclic complex $\boldsymbol{T}$ with $\mathrm{C}_{0}(\boldsymbol{T})=G$. Therefore, $\boldsymbol{T}_{\geq 0} \rightarrow G$ is a projective resolution, so for all $i>0$ one has

$$
\operatorname{Ext}_{R}^{i}(G, Q)=\mathrm{H}^{i}\left(\operatorname{Hom}_{R}\left(\boldsymbol{T}_{\geq 0}, Q\right)\right)=\mathrm{H}^{i}\left(\operatorname{Hom}_{R}(\boldsymbol{T}, Q)\right)=0 .
$$

Lemma 2.3.3. For an exact complex of projectives $\boldsymbol{T}$ the following are equivalent:

(i) $\boldsymbol{T}$ is totally acyclic.

(ii) $\mathrm{C}_{i}(\boldsymbol{T})$ is Gorenstein projective for all $i \in \mathbb{Z}$.

(iii) $\mathrm{C}_{i}(\boldsymbol{T})$ is Gorenstein projective for infinitely many $i \leq 0$.

Proof. The implications (i) $\Longrightarrow$ (ii) $\Longrightarrow$ (iii) are clear, so we only argue (iii) $\Longrightarrow$ (i). Let $Q$ be a projective $R$-module and fix $n \in \mathbb{Z}$. We need to show $\mathrm{H}^{n}\left(\operatorname{Hom}_{R}(\boldsymbol{T}, Q)\right)$ $=0$. Choose an integer $m \geq 1$ so that $n-m$ is small enough for $\mathrm{C}_{n-m}(\boldsymbol{T})$ to be Gorenstein projective. The conclusion follows via 2.3.2;

$$
\mathrm{H}^{n}\left(\operatorname{Hom}_{R}(\boldsymbol{T}, Q)\right)=\operatorname{Ext}_{R}^{m}\left(\mathrm{C}_{n-m}(\boldsymbol{T}), Q\right)=0 .
$$

Lemma 2.3.4. If $G$ is Gorenstein projective and $\boldsymbol{P} \rightarrow G$ is a projective resolution, then there exists a totally acyclic complex $\boldsymbol{T}$ such that $\boldsymbol{T}_{\geq 0}=\boldsymbol{P}$.

Proof. By definition, there is a totally acyclic complex $\boldsymbol{T}^{\prime}$ with $\mathrm{C}_{0}\left(\boldsymbol{T}^{\prime}\right)=G$. Set

$$
T_{i}=\left\{\begin{array}{ll}
T_{i}^{\prime} & \text { for } i<0 ; \\
P_{i} & \text { for } i \geq 0 ;
\end{array} \quad \text { and } \quad \partial_{i}^{\boldsymbol{T}}= \begin{cases}\partial_{i}^{\boldsymbol{T}^{\prime}} & \text { for } i<0 \\
\beta \alpha & \text { for } i=0 ; \\
\partial_{i}^{\boldsymbol{P}} & \text { for } i>0\end{cases}\right.
$$

where $\alpha: P_{0} \rightarrow G$ and $\beta: G \rightarrow T_{-1}^{\prime}$ are the canonical maps. The complex $\boldsymbol{T}$ is exact, $\mathrm{C}_{0}(\boldsymbol{T})=G$ and $\boldsymbol{T}_{<0}=\boldsymbol{T}_{<0}^{\prime}$, so $\boldsymbol{T}$ is totally acyclic by Lemma 2.3.3.

A basic property of Gorenstein projective modules is proved by Holm [20, (2.5)].

2.3.5. The class of Gorenstein projective modules has the following properties:

(1) In every exact sequence $0 \rightarrow G \rightarrow G^{\prime} \rightarrow G^{\prime \prime} \rightarrow 0$ of $R$-modules with $G^{\prime \prime}$ Gorenstein projective, the module $G$ is Gorenstein projective if and only if the module $G^{\prime}$ is Gorenstein projective.

(2) When $G^{\prime} \cong G \oplus G^{\prime \prime}$, the module $G^{\prime}$ is Gorenstein projective if and only if both modules $G$ and $G^{\prime \prime}$ are Gorenstein projectives.

(3) Every projective module is Gorenstein projective.

Let $M$ be a left $R$-module. 
2.3.6. Following Enochs and Jenda [13], we say that $M$ has Gorenstein projective dimension $g$, and write $\operatorname{Gpd}_{R} M=g$, if there exists an exact sequence

$$
0 \rightarrow G_{g} \rightarrow G_{g-1} \rightarrow \cdots \rightarrow G_{1} \rightarrow G_{0} \rightarrow M \rightarrow 0
$$

where $G_{i}$ is Gorenstein projective for $i=0, \ldots, g$, and there is no shorter exact sequence with this property.

The next three results are due to Holm.

2.3.7 ([20, (2.7)]). One has $\operatorname{Gpd}_{R} M \leq n$ if in one, and only if in every exact sequence

$$
0 \rightarrow K_{n} \rightarrow G_{n-1}^{\prime} \rightarrow \cdots \rightarrow G_{1}^{\prime} \rightarrow G_{0}^{\prime} \rightarrow M \rightarrow 0
$$

where all $G_{i}^{\prime}$ are Gorenstein projective, the module $K_{n}$ is Gorenstein projective.

2.3.8 ([20, (2.27)]). One has $\operatorname{Gpd}_{R} M \leq \operatorname{pd}_{R} M$, with equality when $\operatorname{pd}_{R} M$ is finite.

2.3.9 (20, (2.19)]). For every family of $R$-modules $\left(M_{i}\right)_{i \in I}$ one has

$$
\operatorname{Gpd}_{R}\left(\coprod_{i \in I} M_{i}\right)=\sup _{i \in I}\left\{\operatorname{Gpd}_{R} M_{i}\right\} .
$$

2.4. Gorenstein dimension. In this subsection we assume that the ring $R$ is left and right noetherian and that $M$ is a finite $R$-module.

Auslander and Bridger [1] define the Gorenstein dimension G- $\operatorname{dim}_{R} M$, as the supremum of those integers $n$ for which there exists an exact sequence

$$
0 \rightarrow G_{n} \rightarrow \cdots \rightarrow G_{1} \rightarrow G_{0} \rightarrow M \rightarrow 0
$$

where each $G_{i}$ is a finite reflexive $R$-module satisfying

$$
\operatorname{Ext}_{R}^{n}\left(G_{i}, R\right)=0=\operatorname{Ext}_{R}^{n}\left(G_{i}^{*}, R\right) \quad \text { for all } \quad n \geq 1 .
$$

2.4.1. The two notions of Gorenstein dimension agree:

$$
\text { G- } \operatorname{dim}_{R} M=\operatorname{Gpd}_{R} M \text {. }
$$

Indeed, G- $\operatorname{dim}_{R} M=0$ if and only if $\operatorname{Gpd}_{R} M=0$ by [12, (4.2.6)]; one deduces from here that equality holds in general using 2.3.7.

Let $\operatorname{id}_{R} R$ (respectively, $\operatorname{id}_{R^{\circ}} R$ ) denote the injective dimension of $R$ as a left (respectively, right) $R$-module.

2.4.2. Let $n$ be a non-negative integer. The following conditions are equivalent:

(i) $\operatorname{id}_{R} R \leq n$ and $\operatorname{id}_{R^{\circ}} R \leq n$.

(ii) Every $R$-module $M$ satisfies $\operatorname{Gpd}_{R} M \leq n$.

(iii) Every finite $R$-module $M$ satisfies G- $\operatorname{dim}_{R} M \leq n$.

Indeed, (i) implies (ii) by [14, (10.2.14)], (ii) implies (iii) by 2.4.1, and (iii) implies (i) by [6, (3.2)].

Gorenstein dimension has been extensively studied over commutative rings. We quote some results that are used in this paper.

2.4.3. Let $R$ be a commutative ring and $M$ a finite $R$-module.

(1) [1, (4.15)] If $\boldsymbol{p}$ is a prime ideal of $R$, then

$$
\text { G-dim } R_{R_{p}} M_{\boldsymbol{p}} \leq \mathrm{G}-\operatorname{dim}_{R} M .
$$


(2) [1, (4.13.b)] If $R$ is local and G- $\operatorname{dim}_{R} M$ is finite, then

$$
\text { G- } \operatorname{dim}_{R} M+\operatorname{depth}_{R} M=\operatorname{depth} R \text {. }
$$

We use the term Gorenstein ring to denote a noetherian commutative ring $R$, such that the local ring $R_{m}$ has finite injective dimension as a module over itself for every maximal ideal $\boldsymbol{m}$ of $R$.

2.4.4. If $R$ is Gorenstein, then $\operatorname{id}_{R} M=\operatorname{dim} R$; see [8, (3.1.17)] and [14, (3.2.20)].

The next result, due to Auslander and Bridger [1, (4.20)] and Goto 18, Corollary $2]$, gives the reason for the name Gorenstein dimension.

2.4.5. If $R$ is a commutative noetherian ring, then $R$ is Gorenstein if and only if every finite module has finite Gorenstein dimension.

\section{Gorenstein Projective Dimension}

In this section, first we define a notion of Gorenstein projective dimension for complexes which extends naturally the notions of Gorenstein (projective) dimensions, previously defined by several authors; see Corollary [3.6 and Remark 5.4. Second, we prove our main results, Theorems 3.43 .8 and respectively 3.9 which are parallel to the results on projective dimension 1.4.1, 1.4.2 and respectively 1.4.3. and Theorem 3.10 which generalizes 2.4 .5

Let $M$ be a complex of left $R$-modules.

Definition 3.1. The Gorenstein projective dimension of $\boldsymbol{M}$ is defined by

$$
\operatorname{Gpd}_{R} \boldsymbol{M}=\inf \left\{\begin{array}{l|c}
g \in \mathbb{Z} & \begin{array}{c}
\boldsymbol{T} \stackrel{\tau}{\rightarrow} \boldsymbol{P} \stackrel{\pi}{\rightarrow} \boldsymbol{M} \\
\text { is a complete resolution with } \\
\tau_{i}: T_{i} \rightarrow P_{i} \text { bijective for each } i \geq g
\end{array}
\end{array}\right\} .
$$

The next two assertions follow from the definition.

3.2. The complex $\boldsymbol{M}$ is exact if and only if $\operatorname{Gpd}_{R} \boldsymbol{M}=-\infty$.

3.3. For every $i \in \mathbb{Z}$, one has $\operatorname{Gpd}_{R} \Sigma^{i} \boldsymbol{M}=\operatorname{Gpd}_{R} \boldsymbol{M}+i$.

The next theorem can be viewed as an equivalent definition of $\operatorname{Gpd}_{R} \boldsymbol{M}$, expressed in terms of an arbitrary semiprojective resolution of the complex $\boldsymbol{M}$.

Theorem 3.4. For each integer $g$ the following conditions are equivalent:

(i) $\operatorname{Gpd}_{R} \boldsymbol{M} \leq g$.

(ii) $\sup \mathrm{H}(\boldsymbol{M}) \leq g$ and there exists a semiprojective resolution $\boldsymbol{P} \rightarrow \boldsymbol{M}$ such that the module $\mathrm{C}_{g}(\boldsymbol{P})$ is Gorenstein projective.

$\left(\mathrm{ii}^{\prime}\right) \sup \mathrm{H}(\boldsymbol{M}) \leq g$ and for every semiprojective resolution $\boldsymbol{P}^{\prime} \rightarrow \boldsymbol{M}$ the module $\mathrm{C}_{g}\left(\boldsymbol{P}^{\prime}\right)$ is Gorenstein projective.

(iii) $\sup \mathrm{H}(\boldsymbol{M}) \leq g$ and $\Omega_{g}(\boldsymbol{M})$ is Gorenstein projective.

(iv) For every semiprojective resolution $\boldsymbol{P}^{\prime} \rightarrow \boldsymbol{M}$ there exists a surjective complete resolution $\boldsymbol{T}^{\prime} \stackrel{\tau^{\prime}}{\longrightarrow} \boldsymbol{P}^{\prime} \rightarrow \boldsymbol{M}$ such that $\tau_{i}^{\prime}=\mathrm{id}^{T_{i}^{\prime}}$ for all $i \geq g$.

Proof. (i) $\Longrightarrow$ (ii). By hypothesis, there is a complete resolution $\boldsymbol{T} \stackrel{\tau}{\rightarrow} \boldsymbol{P} \stackrel{\pi}{\rightarrow}$ $\boldsymbol{M}$ such that $\tau_{\geq g}: \boldsymbol{T}_{\geq g} \rightarrow \boldsymbol{P}_{\geq g}$ is an isomorphism of complexes. This implies isomorphisms $\mathrm{H}_{i}(\boldsymbol{M}) \cong \mathrm{H}_{i}(\boldsymbol{P})$ for all $i \in \mathbb{Z}, \mathrm{H}_{i}(\boldsymbol{P}) \cong \mathrm{H}_{i}(\boldsymbol{T})$ for all $i>g$, and 
$\mathrm{C}_{g}(\boldsymbol{P}) \cong \mathrm{C}_{g}(\boldsymbol{T})$. Since the complex $\boldsymbol{T}$ is totally acyclic, $\mathrm{H}_{i}(\boldsymbol{T})$ vanishes for each $i \in \mathbb{Z}$ and $\mathrm{C}_{g}(\boldsymbol{T})$ is Gorenstein projective.

(ii) $\Longrightarrow\left(\right.$ ii' $^{\prime}$. Let $\boldsymbol{P}^{\prime} \stackrel{\pi^{\prime}}{\longrightarrow} \boldsymbol{M}$ be a semiprojective resolution. By Proposition 1.3.6. there exist projective modules $Q_{g}$ and $Q_{g}^{\prime}$ such that $\mathrm{C}_{g}(\boldsymbol{P}) \oplus Q_{g}^{\prime} \cong \mathrm{C}_{g}\left(\boldsymbol{P}^{\prime}\right) \oplus Q_{g}$. Thus, $\mathrm{C}_{g}\left(\boldsymbol{P}^{\prime}\right)$ is Gorenstein projective by 2.3.5.

(ii') $\Longrightarrow$ (iii) is clear.

(iii) $\Longrightarrow$ (iv). Let $\boldsymbol{P}^{\prime} \rightarrow \boldsymbol{M}$ be a semiprojective resolution with $\Omega_{g}(\boldsymbol{M})=$ $\mathrm{C}_{g}\left(\boldsymbol{P}^{\prime}\right)$ Gorenstein projective and $\mathrm{H}_{i}\left(\boldsymbol{P}^{\prime}\right)=0$ for all $i>g$. Then $\Sigma^{-g} \boldsymbol{P}_{\geq g}^{\prime} \rightarrow$ $\mathrm{C}_{g}\left(\boldsymbol{P}^{\prime}\right)$ is a projective resolution. Lemma 2.3.4, yields a totally acyclic complex $\boldsymbol{T}^{\prime \prime}$ with $\boldsymbol{T}_{\geq g}^{\prime \prime}=\boldsymbol{P}_{\geq g}^{\prime}$. Applying 2.1.2, we obtain a complete resolution $\boldsymbol{T}^{\prime \prime} \stackrel{\tau^{\prime \prime}}{\longrightarrow} \boldsymbol{P}^{\prime} \rightarrow \boldsymbol{M}$ with $\tau_{i}^{\prime \prime}=\mathrm{id}^{T_{i}^{\prime \prime}}$ for all $i \geq g$, and $\mathrm{C}_{g}\left(\boldsymbol{T}^{\prime \prime}\right) \cong \mathrm{C}_{g}\left(\boldsymbol{P}^{\prime}\right)$. From 2.2.2 we get a surjective complete resolution $\boldsymbol{T}^{\prime} \stackrel{\tau^{\prime}}{\longrightarrow} \boldsymbol{P}^{\prime} \stackrel{\pi^{\prime}}{\longrightarrow} \boldsymbol{M}$ with the desired properties.

(iv) $\Longrightarrow$ (i) is clear.

Corollary 3.5. For every family of complexes of $R$-modules $\left(\boldsymbol{M}_{i}\right)_{i \in I}$ one has

$$
\operatorname{Gpd}_{R}\left(\coprod_{i \in I} \boldsymbol{M}_{i}\right)=\sup _{i \in I}\left\{\operatorname{Gpd}_{R} \boldsymbol{M}_{i}\right\} .
$$

Proof. Choose for each $i \in I$ a semiprojective resolution $\boldsymbol{P}_{i} \rightarrow \boldsymbol{M}_{i}$ and set $\boldsymbol{P}=\coprod_{i \in I} \boldsymbol{P}_{i}$. As $\boldsymbol{P} \rightarrow \coprod_{i \in I} \boldsymbol{M}_{i}$ is a semiprojective resolution and $\mathrm{C}_{n}(\boldsymbol{P})=$ $\coprod_{i \in I} \mathrm{C}_{n}(\boldsymbol{P})$ for each $n \in \mathbb{Z}$, the assertion follows from the theorem and 2.3.9.

We reconcile the two notions of Gorenstein projective dimension for modules.

Corollary 3.6. If $M$ is an R-module and $g$ (respectively, $g^{\prime}$ ) denote the Gorenstein projective dimension of $M$ in the sense of 2.3.6 (respectively, of [3.1), then $g=g^{\prime}$.

Proof. Choose a classical projective resolution $\boldsymbol{P} \rightarrow M$, and let $h$ be the smallest integer $n$ such that $\mathrm{C}_{n}(\boldsymbol{P})$ is Gorenstein projective. As each $P_{i}$ is Gorenstein projective, 2.3.7 implies $h=g$. On the other hand, as $\boldsymbol{P} \rightarrow M$ is a semiprojective resolution by 1.3.5. Theorem 3.4 yields $h=g^{\prime}$.

Another consequence of the Theorem 3.4 is an extension to complexes of 2.3.8,

Theorem 3.7. One has $\operatorname{Gpd}_{R} \boldsymbol{M} \leq \operatorname{pd}_{R} \boldsymbol{M}$, with equality if $\operatorname{pd}_{R} \boldsymbol{M}$ is finite.

Proof. Set $p=\operatorname{pd}_{R} \boldsymbol{M}$ and $g=\operatorname{Gpd}_{R} \boldsymbol{M}$. There is nothing to prove if $p=\infty$. If $p=-\infty$, then, by definition $\mathrm{H}(\boldsymbol{M})=0$. Therefore, by 3.2. $\operatorname{Gpd}_{R} \boldsymbol{M}=-\infty$. We may assume that $p$ is finite.

By 1.4.1, $\mathrm{H}_{i}(\boldsymbol{M})=0$ for $i>p$ and there exists a semiprojective resolution $\boldsymbol{P} \rightarrow \boldsymbol{M}$ in which the module $\mathrm{C}_{p}(\boldsymbol{P})$ is projective, hence Gorenstein projective. Applying Theorem 3.4, we obtain $g \leq p$.

Suppose $g<p$. By definition, there exists a semiprojective resolution $\boldsymbol{P}^{\prime} \rightarrow \boldsymbol{M}$ such that $P_{i}^{\prime}=0$ for all $i>p$. By Theorem 3.4. $\mathrm{C}_{g}\left(\boldsymbol{P}^{\prime}\right)$ is Gorenstein projective and $\mathrm{H}_{i}(\boldsymbol{M})=0$ for all $i>g$, in particular, $\mathrm{H}_{i}\left(\boldsymbol{P}^{\prime}\right)=0$ for all $i>g$. Therefore, there exists an exact sequence of $R$-modules

$$
0 \rightarrow P_{p}^{\prime} \rightarrow \cdots \rightarrow P_{g}^{\prime} \rightarrow \mathrm{C}_{g}\left(\boldsymbol{P}^{\prime}\right) \rightarrow 0 .
$$

Thus, the Gorenstein projective module $\mathrm{C}_{g}\left(\boldsymbol{P}^{\prime}\right)$ has finite projective dimension, so it is projective by 2.3.8. Now1.4.1 implies $p \leq g$, which contradicts our assumption. Hence $p=g$, as desired. 
The next theorem extends to complexes a result of Holm [20, (2.20)]. While formally similar to the characterization of projective dimension in terms of vanishing of appropriate Ext groups (see 1.4.2), it differs in a significant aspect: it is restricted to complexes of finite Gorenstein projective dimension.

Theorem 3.8. If $\operatorname{Gpd}_{R} \boldsymbol{M}=g<\infty$, then there are equalities

$$
\begin{aligned}
\operatorname{Gpd}_{R} \boldsymbol{M} & =\sup \left\{\begin{array}{l|c}
n \in \mathbb{Z} & \begin{array}{c}
\operatorname{Ext}_{R}^{n}(\boldsymbol{M}, Q) \neq 0 \\
\text { for some projective module } Q
\end{array}
\end{array}\right\} \\
& =\sup \left\{\begin{array}{l|c}
n \in \mathbb{Z} & \begin{array}{c}
\operatorname{Ext}_{R}^{n-\inf \mathrm{H}(\boldsymbol{N})}(\boldsymbol{M}, \boldsymbol{N}) \neq 0 \\
\text { for some complex } \boldsymbol{N} \text { with } \\
\operatorname{pd}_{R} \boldsymbol{N}<\infty \text { and inf } \mathrm{H}(\boldsymbol{N})>-\infty
\end{array}
\end{array}\right\} .
\end{aligned}
$$

Proof. When $\operatorname{Gpd}_{R} \boldsymbol{M}=-\infty$, we use 3.2. Suppose that $g$ is finite. By definition, there is a complete resolution $\boldsymbol{T} \stackrel{\tau}{\rightarrow} \boldsymbol{P} \stackrel{\pi}{\rightarrow} \boldsymbol{M}$ such that $\tau_{\geq g}: \boldsymbol{T}_{\geq g} \rightarrow \boldsymbol{P}_{\geq g}$ is an isomorphism. Let $h$ (respectively, $h^{\prime}$ ) denote the number on the right-hand side of the first (respectively, second) equality of the theorem.

First, we show $g \geq h^{\prime}$. By 1.3 .4 and 1.4 .1 choose a semiprojective resolution $\boldsymbol{Q} \rightarrow \boldsymbol{N}$ with $\inf \boldsymbol{Q}=\inf \mathrm{H}(\boldsymbol{N})$ and $\sup \boldsymbol{Q}<\infty$. For all $i>g-\inf \mathrm{H}(\boldsymbol{N})$ we have

$$
\begin{aligned}
\operatorname{Ext}_{R}^{i}(\boldsymbol{M}, \boldsymbol{N}) & =\mathrm{H}^{i}\left(\operatorname{Hom}_{R}(\boldsymbol{P}, \boldsymbol{N})\right) \\
& \cong \mathrm{H}^{i}\left(\operatorname{Hom}_{R}(\boldsymbol{P}, \boldsymbol{Q})\right) \\
& =\mathrm{H}^{i}\left(\operatorname{Hom}_{R}(\boldsymbol{T}, \boldsymbol{Q})\right) \\
& =0 .
\end{aligned}
$$

Indeed, the first equality holds by definition; the isomorphism is due to the semiprojectivity of $\boldsymbol{P}$; for the third equality we use the isomorphism $\boldsymbol{P}_{\geq g} \cong T_{\geq g}$ and the choice of $i$; the last equality follows from 2.1.3.

The inequality $h^{\prime} \geq h$ is obvious, so it remains to prove $h \geq g$.

By way of contradiction, assume $h<g$. Then the sequence

$$
\begin{aligned}
& \operatorname{Hom}_{R}\left(P_{g-1}, T_{g-1}\right) \stackrel{\operatorname{Hom}_{R}\left(\partial_{g}^{P}, T_{g-1}\right)}{\longrightarrow} \operatorname{Hom}_{R}\left(P_{g}, T_{g-1}\right) \\
& \stackrel{\operatorname{Hom}_{R}\left(\partial_{g+1}^{P}, T_{g-1}\right)}{\longrightarrow} \operatorname{Hom}_{R}\left(P_{g+1}, T_{g-1}\right)
\end{aligned}
$$

is exact. Let $\alpha: P_{g} \cong T_{g} \rightarrow T_{g-1}$ be the canonical map. Since $\operatorname{Hom}_{R}\left(\partial_{g+1}^{\boldsymbol{P}}, T_{g-1}\right)(\alpha)$ $=0$, there exists a map $\beta: P_{g-1} \rightarrow T_{g-1}$ such that $\beta \partial_{g}^{P}=\alpha$. It is easy to see that the pair $\left(\boldsymbol{T}^{\prime}, \partial^{\boldsymbol{T}^{\prime}}\right)$ given by

$$
T_{i}^{\prime}=\left\{\begin{array}{ll}
T_{i} & \text { for } i \neq g-1 ; \\
P_{g-1} & \text { for } i=g-1 ;
\end{array} \quad \text { and } \quad \partial_{i}^{\boldsymbol{T}^{\prime}}= \begin{cases}\partial_{i}^{\boldsymbol{T}} & \text { for } i \neq g, g-1 \\
\tau_{g-1} \partial_{g}^{\boldsymbol{T}} & \text { for } i=g \\
\partial_{g-1}^{\boldsymbol{T}} \beta & \text { for } i=g-1\end{cases}\right.
$$

is an exact complex. Since each module $T_{i}^{\prime}$ is projective and $\mathrm{C}_{i}\left(\boldsymbol{T}^{\prime}\right)$ is Gorenstein projective for all $i \ll 0, \boldsymbol{T}^{\prime}$ is totally acyclic by 2.3.3. Thus, we obtain a complete resolution $\boldsymbol{T}^{\prime} \stackrel{\tau^{\prime}}{\longrightarrow} \boldsymbol{P} \stackrel{\pi}{\longrightarrow} \boldsymbol{M}$ with $\tau_{i}^{\prime}$ bijective for each $i \geq g-1$. Therefore, we get $\operatorname{Gpd}_{R} \boldsymbol{M} \leq g-1$; this is a contradiction, hence $h=g$. 
The next result extends [6, (4.9.2)] to complexes and gives a parallel to 1.4.3.

Theorem 3.9. Let $0 \rightarrow \boldsymbol{M} \rightarrow \boldsymbol{M}^{\prime} \rightarrow \boldsymbol{M}^{\prime \prime} \rightarrow 0$ be an exact sequence of complexes.

(1) If two complexes have finite Gorenstein projective dimension, then so does the third.

(2) There is an inequality

$$
\begin{aligned}
& \operatorname{Gpd}_{R} \boldsymbol{M}^{\prime} \leq \max \left(\operatorname{Gpd}_{R} \boldsymbol{M}, \operatorname{Gpd}_{R} \boldsymbol{M}^{\prime \prime}\right) \\
& \text { and equality holds, except possibly when } \operatorname{Gpd}_{R} \boldsymbol{M}^{\prime \prime}=\operatorname{Gpd}_{R} \boldsymbol{M}+1 .
\end{aligned}
$$

Proof. (1) By Proposition 1.3 .8 there exists a commutative diagram

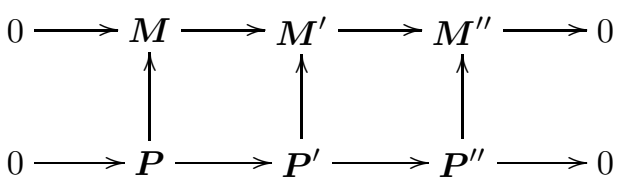

where the columns are semiprojective resolutions and the bottom short sequence is exact. The exact sequences of homology groups shows that if two of the semiprojective complexes have bounded above cohomology, then so has the third. Using Theorem 3.4, choose $n$ so that

$$
\mathrm{H}_{i}(\boldsymbol{P})=\mathrm{H}_{i}\left(\boldsymbol{P}^{\prime}\right)=\mathrm{H}_{i}\left(\boldsymbol{P}^{\prime \prime}\right)=0 \quad \text { for all } \quad i \geq n .
$$

For all $i \geq n$ the sequence of modules

$$
0 \rightarrow \mathrm{C}_{i}(\boldsymbol{P}) \rightarrow \mathrm{C}_{i}\left(\boldsymbol{P}^{\prime}\right) \rightarrow \mathrm{C}_{i}\left(\boldsymbol{P}^{\prime \prime}\right) \rightarrow 0
$$

is then exact. If $\mathrm{C}_{i}(\boldsymbol{P})$ (respectively $\mathrm{C}_{i}\left(\boldsymbol{P}^{\prime}\right)$ ) and $\mathrm{C}_{i}\left(\boldsymbol{P}^{\prime \prime}\right.$ ) are Gorenstein projective, then 2.3.5 shows that so is $\mathrm{C}_{i}\left(\boldsymbol{P}^{\prime}\right)$ (respectively $\mathrm{C}_{i}(\boldsymbol{P})$ ). If $\mathrm{C}_{i}(\boldsymbol{P})$ and $\mathrm{C}_{i}\left(\boldsymbol{P}^{\prime}\right)$ are Gorenstein projectives, then $\operatorname{Gpd}_{R} \mathrm{C}_{i}\left(\boldsymbol{P}^{\prime \prime}\right) \leq 1$; hence by 2.3.7. $\mathrm{C}_{i+1}\left(\boldsymbol{P}^{\prime \prime}\right)$ is Gorenstein projective. Applying Theorem [3.4 we obtain the desired conclusion.

(2) By (1) we may assume that all complexes have finite Gorenstein projective dimension. The conclusion then follows by considering the exact sequences of groups $\operatorname{Ext}_{R}(-, Q)$, induced by the short exact sequence from hypothesis, where $Q$ varies over all projective modules, and applying Theorem 3.8 .

The next result extends 2.4.2 It characterizes Gorenstein rings in terms of the finiteness of the Gorenstein projective dimension of complexes.

Theorem 3.10. If $R$ is a left and right noetherian ring and $n$ is a non-negative integer, then the following are equivalent:

(i) $\operatorname{id}_{R} R \leq n$ and $\operatorname{id}_{R^{\circ}} R \leq n$.

(ii) Every complex $\boldsymbol{M}$ of left $R$-modules or right $R$-modules satisfies

$$
\operatorname{Gpd}_{R} \boldsymbol{M} \leq \sup \mathrm{H}(\boldsymbol{M})+n .
$$

Proof. (ii) $\Longrightarrow$ (i) holds by 2.4.2,

To prove (i) $\Longrightarrow$ (ii) it is enough to consider left modules since all the results used in the argument hold also for right modules.

We may assume $\sup \mathrm{H}(\boldsymbol{M})=t<\infty$. Set $g=t+n$ and choose a semiprojective resolution $\boldsymbol{P} \rightarrow \boldsymbol{M}$. By 2.4.2, every module has Gorenstein projective dimension at most $n$, in particular, $\operatorname{Gpd}_{R} \mathrm{C}_{t}(\boldsymbol{P}) \leq n$. From the exact sequence

$$
0 \rightarrow \mathrm{C}_{g}(\boldsymbol{P}) \rightarrow P_{g-1} \rightarrow \cdots \rightarrow P_{t} \rightarrow \mathrm{C}_{t}(\boldsymbol{P}) \rightarrow 0
$$


one concludes that the module $\mathrm{C}_{g}(\boldsymbol{P})$ is Gorenstein projective; see2.3.7 Therefore, Theorem 3.4 yields $\operatorname{Gpd}_{R} \boldsymbol{M} \leq g$.

Another variation on the same theme was pointed out by Avramov and Iyengar.

Theorem 3.11. Let $R$ be a commutative noetherian ring.

(1) $R$ is Gorenstein and $\operatorname{dim} R$ is finite if and only if $\operatorname{Gpd}_{R} \boldsymbol{M}$ is finite for every complex of $R$-modules with $\mathrm{H}(\boldsymbol{M})$ bounded above.

(2) $R$ is Gorenstein if and only if $\operatorname{Gpd}_{R} \boldsymbol{M}$ is finite for every complex $\boldsymbol{M}$ of $R$ modules with $\mathrm{H}(\boldsymbol{M})$ bounded and $\mathrm{H}_{i}(\boldsymbol{M})$ finitely generated for each $i \in \mathbb{Z}$.

Proof. (1) If $R$ is Gorenstein and $\operatorname{dim} R$ is finite, then $\operatorname{id}_{R} R$ is finite by 2.4.4, so $\operatorname{Gpd}_{R} \boldsymbol{M}<\infty$ for every complex with $\sup \mathrm{H}(\boldsymbol{M})<\infty$ by Theorem 3.10.

Assume now that $\operatorname{Gpd}_{R} M$ is finite for every $R$-module $M$. The $\operatorname{ring} R$ is then Gorenstein by 2.4.1 and 2.4.5. Set $M=\coprod_{m} R / \boldsymbol{m}$, where the sum runs over all maximal ideals of $R$. For every maximal ideal $\boldsymbol{m}$ of $R$ we then obtain

$$
\operatorname{dim} R_{\boldsymbol{m}}=\operatorname{Gpd}_{R_{\boldsymbol{m}}}\left(R_{\boldsymbol{m}} / \boldsymbol{m} R_{\boldsymbol{m}}\right) \leq \operatorname{Gpd}_{R}(R / \boldsymbol{m}) \leq \operatorname{Gpd}_{R} M
$$

where the first two relations are obtained by using 2.4.1 with 2.4.3, and the third one comes from 2.3.9. As a consequence, we get $\operatorname{dim} R \leq \operatorname{Gpd}_{R} M<\infty$.

(2) If $\operatorname{Gpd}_{R} M<\infty$ for every finite $R$-module, then 2.4.1 and 2.4.5 imply that $R$ is Gorenstein, as above.

Assume now that $R$ is Gorenstein and $\boldsymbol{M}$ is a complex with $\mathrm{H}(\boldsymbol{M})$ bounded and $\mathrm{H}_{i}(\boldsymbol{M})$ finitely generated for each $i \in \mathbb{Z}$. By 1.3 .4 there exists a semiprojective resolution $\boldsymbol{P} \rightarrow \boldsymbol{M}$, with $P_{i}$ finitely generated projective for each $i$. If $\sup \mathrm{H}(\boldsymbol{M})=$ $t$, then 2.4.5 and 2.4.1 yield $\operatorname{Gpd}_{R} \mathrm{C}_{t}(\boldsymbol{P})=n<\infty$. Setting $g=t+n$, from the exact sequence in the proof of Theorem 3.10 we obtain $\operatorname{Gpd}_{R} M \leq g$.

\section{TATE COHOMOlOGY}

Tate cohomology for modules over finite groups was originally introduced, through complete resolutions, by Tate; see [10, Ch. XII]. It was extended to groups of virtually finite cohomological dimension by Farrell [15] and over groups admitting complete cohomological functors by Gedrich and Gruenberg [16]. In various contexts cohomology theories based on complete resolutions are constructed by Buchweitz [9], Cornick and Kropholler [11, Avramov and Martsinkovsky.

In this section, we define and study a Tate cohomology theory for complexes of left modules over associative rings. Our definition is modeled on the one of Avramov and Martsinkovsky [6].

We let $\mathcal{C}_{\mathcal{G P}}$ denote the class of complexes of finite Gorenstein projective dimension, and we fix a complete resolution for each complex in $\mathcal{C}_{\mathcal{G P}}$.

Throughout this section $\boldsymbol{M}$ denotes a complex in $\mathcal{C}_{\mathcal{G P}}, \boldsymbol{T} \stackrel{\tau}{\rightarrow} \boldsymbol{P} \stackrel{\pi}{\rightarrow} \boldsymbol{M}$ as its chosen resolution, and let $\boldsymbol{N}$ denote an arbitrary complex. For each $n \in \mathbb{Z}$, the $n^{\text {th }}$ Tate cohomology group is defined by

$$
\operatorname{Ext}_{R}^{n}(\boldsymbol{M}, \boldsymbol{N})=\mathrm{H}^{n}\left(\operatorname{Hom}_{R}(\boldsymbol{T}, \boldsymbol{N})\right) .
$$

The morphism

$$
\operatorname{Hom}_{R}(\tau, \boldsymbol{N}): \operatorname{Hom}_{R}(\boldsymbol{P}, \boldsymbol{N}) \rightarrow \operatorname{Hom}_{R}(\boldsymbol{T}, \boldsymbol{N})
$$

induces for every $n \in \mathbb{Z}$ a homomorphism of abelian groups

$$
\hat{\varepsilon}_{R}^{n}(\boldsymbol{M}, \boldsymbol{N}): \operatorname{Ext}_{R}^{n}(\boldsymbol{M}, \boldsymbol{N}) \rightarrow \operatorname{Ext}_{R}^{n}(\boldsymbol{M}, \boldsymbol{N}) .
$$


Proposition-Definition 4.1. (1) The assignment $(\boldsymbol{M}, \boldsymbol{N}) \mapsto \operatorname{Ext}_{R}^{n}(\boldsymbol{M}, \boldsymbol{N})$ defines a functor

$$
\mathrm{Ext}_{R}^{n}(-,-):\left(\mathcal{C}_{\mathcal{G P}}\right)^{o p} \times \mathcal{C} \rightarrow \mathcal{M}(\mathbb{Z})
$$

which is independent of choices of resolutions and liftings.

(2) The maps $\hat{\varepsilon}_{R}^{n}(\boldsymbol{M}, \boldsymbol{N})$ yield a morphism of functors

$$
\hat{\varepsilon}_{R}^{n}(-,-): \operatorname{Ext}_{R}^{n}(-,-) \rightarrow \operatorname{Ext}_{R}^{n}(-,-)
$$

which is independent of choices of resolutions and liftings.

Proof. The naturality of $\hat{E x t}_{R}^{n}(-,-)$ and of $\hat{\varepsilon}_{R}^{n}(-,-)$ follows from the first part of Proposition 2.2.4 applied to the chosen complete resolutions of $\boldsymbol{M}$ and $\boldsymbol{M}^{\prime}$. Their independence from the choices of resolutions and liftings follows from the last part of the same proposition.

The results below follow from the definition of Tate cohomology.

4.2. If $M$ and $N$ are modules with $\operatorname{Gpd}_{R} M=g<\infty$, then the natural map $\hat{\varepsilon}_{R}(M, N)$ is equal to 0 for $n<0$ and is bijective for $n>g$.

4.3. For any finite set of complexes $\left\{\boldsymbol{M}^{i}\right\}_{i \in I}$ of finite Gorenstein projective dimension and any family of complexes $\left\{\boldsymbol{N}^{j}\right\}_{j \in J}$, there is a natural isomorphism

$$
\operatorname{Ext}_{R}^{n}\left(\coprod_{i \in I} M^{i}, \prod_{j \in J} \boldsymbol{N}^{j}\right) \cong \prod_{(i, j) \in I \times J} \operatorname{Ext}_{R}^{n}\left(M^{i}, \boldsymbol{N}^{j}\right) \text { for each } n \in \mathbb{Z} .
$$

4.4. There is an isomorphism

$$
\mathrm{Ext}_{R}^{n}\left(\Sigma^{j} \boldsymbol{M},-\right) \cong \operatorname{Ext}_{R}^{n-j}(\boldsymbol{M},-) \text { for all } n, j \in \mathbb{Z} .
$$

Unlike ordinary Ext functors, Tate cohomology is rigid. In the case when $R$ is noetherian and $M$ is a finite module, the theorem below specializes to [6, (5.9)].

Theorem 4.5. Let $\boldsymbol{M}$ be a complex with $\operatorname{Gpd}_{R} \boldsymbol{M}<\infty$. The following properties are equivalent:

(i) $\operatorname{pd}_{R} \boldsymbol{M}<\infty$.

(ii) $\operatorname{Ext}_{R}^{i}(\boldsymbol{M},-)=0$ for some $i \in \mathbb{Z}$.

(ii') $\operatorname{Ext}_{R}^{i}(\boldsymbol{M},-)=0$ for all $i \in \mathbb{Z}$.

When $\inf \boldsymbol{M}>-\infty$, the properties above are also equivalent to the following:

(iii) $\operatorname{Ext}_{R}^{i}(-, M)=0$ for some $i \in \mathbb{Z}$.

(iii') $\operatorname{Ext}_{R}^{i}(-, M)=0$ for all $i \in \mathbb{Z}$.

(iv) $\mathrm{Ext}_{R}^{0}(\boldsymbol{M}, \boldsymbol{M})=0$.

Proof. (i) $\Longrightarrow\left(\right.$ ii' $^{\prime}$. If $\operatorname{pd}_{R} \boldsymbol{M}<\infty$, then $\mathbf{0} \stackrel{\tau}{\rightarrow} \boldsymbol{P} \stackrel{\pi}{\rightarrow} \boldsymbol{M}$ is a complete resolution for any semiprojective resolution $\boldsymbol{P} \stackrel{\pi}{\rightarrow} \boldsymbol{M}$, so the group $\operatorname{Ext}_{R}^{n}(\boldsymbol{M},-)=$ $\mathrm{H}^{n}\left(\operatorname{Hom}_{R}(\mathbf{0},-)\right)$ vanishes for all $n \in \mathbb{Z}$.

$\left(\right.$ ii $\left.^{\prime}\right) \Longrightarrow$ (ii) is clear.

(ii) $\Longrightarrow$ (i). Choose a complete resolution $\boldsymbol{T} \stackrel{\tau}{\rightarrow} \boldsymbol{P} \stackrel{\pi}{\rightarrow} \boldsymbol{M}$. Set $G=\mathrm{C}_{i}(\boldsymbol{T})$ and let $\iota: G \rightarrow T_{i-1}$ be the canonical injection. Since $\operatorname{Ext}_{R}^{i}(\boldsymbol{M},-)=0$, the map 
$\alpha=\operatorname{Hom}_{R}(\iota, G)$ in the commutative diagram

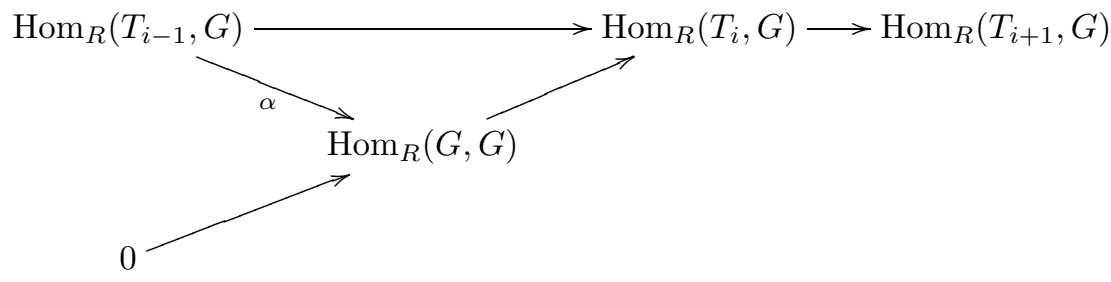

is surjective. This means that $\iota$ splits, so $G$ is projective. Induction on $j$ shows that $\mathrm{C}_{j}(\boldsymbol{T})$ is projective for all $j \geq i$. By definition, $\mathrm{C}_{j}(\boldsymbol{P}) \cong \mathrm{C}_{j}(\boldsymbol{T})$ for all $j \geq \operatorname{Gpd}_{R} \boldsymbol{M}$, so $\mathrm{C}_{j}(\boldsymbol{P})$ is projective for all $j \geq \max \left\{i, \operatorname{Gpd}_{R} \boldsymbol{M}\right\}$. From 1.4.1 we get $\operatorname{pd}_{R} \boldsymbol{M}<\infty$.

For the rest of the proof we assume inf $\boldsymbol{M}>-\infty$.

(i) $\Longrightarrow$ (iii'). By 1.3 .4 and 1.4 .1 choose a bounded semiprojective resolution $\boldsymbol{P} \rightarrow \boldsymbol{M}$. Now, apply 1.1 .6 and 2.1.3.

$\left(\right.$ iii $\left.^{\prime}\right) \Longrightarrow$ (iii) is clear.

(iii) $\Longrightarrow$ (iv) follows from the isomorphism $\operatorname{Ext}_{R}^{i}\left(\Sigma^{i} \boldsymbol{M}, \boldsymbol{M}\right) \cong \operatorname{Ext}_{R}^{0}(\boldsymbol{M}, \boldsymbol{M})$.

(iv) $\Longrightarrow$ (i). By 1.3.4 choose a bounded below semiprojective resolution $\boldsymbol{P} \rightarrow \boldsymbol{M}$ and by Theorem 3.4 choose a complete resolution $\boldsymbol{T} \stackrel{\tau}{\rightarrow} \boldsymbol{P} \stackrel{\pi}{\rightarrow} \boldsymbol{M}$ with $\tau_{j}=\mathrm{id}^{T_{j}}$ for all $j \gg 0$. By 1.1.6 we get $\operatorname{Ext}_{R}^{0}(\boldsymbol{M}, \boldsymbol{M}) \cong \mathrm{H}^{0} \operatorname{Hom}_{R}(\boldsymbol{T}, \boldsymbol{P})=0$. As $\tau$ is in $\mathrm{Z}^{0}\left(\operatorname{Hom}_{R}(\boldsymbol{T}, \boldsymbol{P})\right)$, there exists a $\sigma \in \operatorname{Hom}_{R}(\boldsymbol{T}, \boldsymbol{P})_{1}$ such that $\partial(\sigma)=\tau$ in $\operatorname{Hom}_{R}(\boldsymbol{T}, \boldsymbol{P})$. Since $\tau_{j}=\mathrm{id}^{T_{j}}$ for all $j \gg 0$, we then have

$$
\sigma_{j-1} \partial_{j}^{\boldsymbol{T}}+\partial_{j+1}^{\boldsymbol{P}} \sigma_{j}=\mathrm{id}^{\boldsymbol{T}_{j}} \quad \text { for all } j \gg 0
$$

Since the complex $\boldsymbol{T}$ is exact, we obtain, in particular,

$$
\partial_{j+1}^{\boldsymbol{T}} \sigma_{j}(x)=x \quad \text { for all } \quad x \in \operatorname{Im}\left(\partial_{j+1}^{\boldsymbol{T}}\right) \quad \text { and all } \quad j \gg 0 .
$$

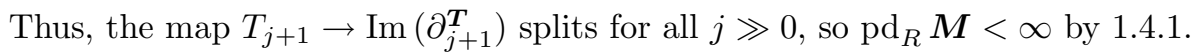

There exist long exact sequences of Tate cohomology groups associated to short exact sequences of complexes in either argument.

The proof of the next result parallels that of $[6,(5.4)]$ and is omitted.

Proposition 4.6. For each complex $\boldsymbol{M}$ with $\operatorname{Gpd}_{R} \boldsymbol{M}<\infty$ and each exact sequence

$$
N_{\bullet}=0 \rightarrow N \stackrel{\vartheta}{\rightarrow} N^{\prime} \stackrel{\vartheta^{\prime}}{\rightarrow} N^{\prime \prime} \rightarrow 0
$$

of complexes of $R$-modules there exist natural in $\boldsymbol{M}$ and $\boldsymbol{N}$. homomorphisms $\hat{\partial}_{R}^{n}\left(\boldsymbol{M}, \boldsymbol{N}_{\bullet}\right)$, such that the sequence below is exact

$$
\begin{aligned}
& \cdots \longrightarrow \operatorname{Ext}_{R}^{n}(\boldsymbol{M}, \boldsymbol{N}) \stackrel{\mathrm{Ext}_{R}^{n}(\boldsymbol{M}, \vartheta)}{\longrightarrow} \operatorname{Ext}_{R}^{n}\left(\boldsymbol{M}, \boldsymbol{N}^{\prime}\right) \stackrel{\mathrm{Ext}_{R}^{n}\left(\boldsymbol{M}, \vartheta^{\prime}\right)}{\longrightarrow} \operatorname{Ext}_{R}^{n}\left(\boldsymbol{M}, \boldsymbol{N}^{\prime \prime}\right) \\
& \stackrel{\hat{\delta}_{R}^{n}\left(\boldsymbol{M}, \boldsymbol{N}_{\bullet}\right)}{\longrightarrow} \mathrm{Ext}_{R}^{n+1}(\boldsymbol{M}, \boldsymbol{N}) \longrightarrow \quad \cdots
\end{aligned}
$$

and for each $n \in \mathbb{Z}$ there is an equality

$$
\hat{\partial}_{R}^{n}\left(\boldsymbol{M}, \boldsymbol{N}_{\bullet}\right) \circ \hat{\varepsilon}_{R}^{n}\left(\boldsymbol{M}, \boldsymbol{N}^{\prime \prime}\right)=\hat{\varepsilon}_{R}^{n+1}(\boldsymbol{M}, \boldsymbol{N}) \circ \partial_{R}^{n}\left(\boldsymbol{M}, \boldsymbol{N}_{\bullet}\right) .
$$


To establish the existence of a long exact sequence in the first argument, one needs a Horseshoe Lemma result for complete resolutions. In the case of modules, such a result is proved in [6, (5.5)]. Different arguments are needed to establish it for complexes, so a complete proof is given.

Proposition 4.7. If $0 \rightarrow M \stackrel{\mu}{\rightarrow} \boldsymbol{M}^{\prime} \stackrel{\mu^{\prime}}{\rightarrow} \boldsymbol{M}^{\prime \prime} \rightarrow 0$ is an exact sequence of complexes of finite Gorenstein projective dimension, then there exists a commutative diagram with exact rows

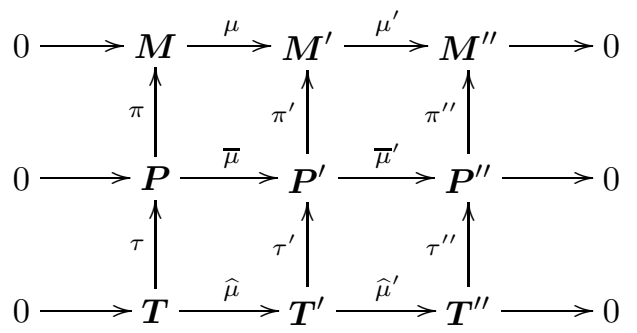

whose columns are surjective complete resolutions.

Proof. Set $g=\max \left(\operatorname{Gpd}_{R} \boldsymbol{M}, \operatorname{Gpd}_{R} \boldsymbol{M}^{\prime \prime}\right)$. Proposition 1.3 .8 and Theorem 3.4 provide all complexes and maps from the diagram (*), except the complex $\boldsymbol{T}^{\prime}$ and the homomorphisms into and out of it. By Theorem 3.4 we can suppose that

$$
\tau_{i}=\mathrm{id}^{T_{i}} \text { and } \tau_{i}^{\prime \prime}=\mathrm{id}^{T_{i}^{\prime \prime}} \quad \text { for all } i \geq g
$$

The rest of the proof proceeds in two steps.

Step 1 . There exists an exact sequence $0 \rightarrow \boldsymbol{T} \rightarrow \boldsymbol{T}^{\prime} \rightarrow \boldsymbol{T}^{\prime \prime} \rightarrow 0$ of complexes of $R$-modules, where $\boldsymbol{T}^{\prime}$ is totally acyclic with $\boldsymbol{T}_{\geq g}^{\prime}=\boldsymbol{P}_{\geq g}^{\prime}$.

We start the proof of this assertion by remarking that any complex $\boldsymbol{T}^{\prime}$ appearing in an exact sequence as above is necessarily totally acyclic. Indeed, acyclicity and the projectivity of each $T_{i}$ are clear. By 2.3.5. for each $i \in \mathbb{Z}$ the exact sequence

$$
0 \rightarrow \mathrm{C}_{i}(\boldsymbol{T}) \rightarrow \mathrm{C}_{i}\left(\boldsymbol{T}^{\prime}\right) \rightarrow \mathrm{C}_{i}\left(\boldsymbol{T}^{\prime \prime}\right) \rightarrow 0
$$

implies $\mathrm{C}_{i}\left(\boldsymbol{T}^{\prime}\right)$ is Gorenstein projective, so $\boldsymbol{T}^{\prime}$ is totally acyclic by Lemma 2.3 .3 .

Next, we describe the construction of the exact sequence in Step 1. Theorem 3.9 gives $\operatorname{Gpd}_{R} \boldsymbol{M}^{\prime} \leq g$, so we obtain

$$
\mathrm{H}_{i}(\boldsymbol{P})=\mathrm{H}_{i}\left(\boldsymbol{P}^{\prime}\right)=\mathrm{H}_{i}\left(\boldsymbol{P}^{\prime \prime}\right)=0 \quad \text { for all } i>g .
$$

Using Theorem 3.4 we get an exact sequence of Gorenstein projective modules

$$
0 \rightarrow \mathrm{C}_{i}(\boldsymbol{P}) \rightarrow \mathrm{C}_{i}\left(\boldsymbol{P}^{\prime}\right) \rightarrow \mathrm{C}_{i}\left(\boldsymbol{P}^{\prime \prime}\right) \rightarrow 0 \quad \text { for all } i>g
$$


Set $T_{i}^{\prime}=P_{i}^{\prime}$ for all $i \geq g$ and $\partial_{i}^{T^{\prime}}=\partial_{i}^{P^{\prime}}$ for all $i>g$, and let $j$ be an integer such that $j<g$. By descending induction, we may assume that a commutative diagram

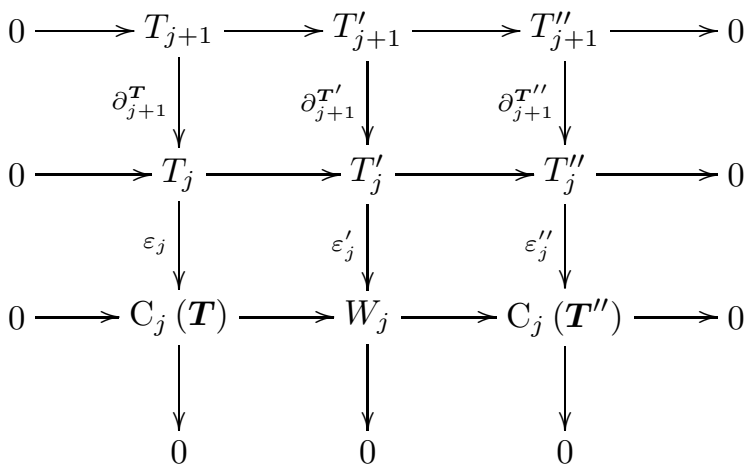

with exact rows and columns was constructed. By 2.3.2 the functor $\operatorname{Hom}_{R}\left(-, T_{j-1}\right)$ transforms the bottom exact sequence into a short exact sequence

$$
0 \rightarrow \operatorname{Hom}_{R}\left(\mathrm{C}_{j}\left(\boldsymbol{T}^{\prime \prime}\right), T_{j-1}\right) \rightarrow \operatorname{Hom}_{R}\left(W_{j}, T_{j-1}\right) \rightarrow \operatorname{Hom}_{R}\left(\mathrm{C}_{j}(\boldsymbol{T}), T_{j-1}\right) \rightarrow 0 .
$$

Therefore, there exists a map $\beta: W_{j} \rightarrow T_{j-1}$ such that $\beta \alpha=\iota_{j}$, where $\iota_{j}: \mathrm{C}_{j}(\boldsymbol{T}) \rightarrow$ $T_{j-1}$ is the canonical injection; see the diagram below. Setting

$$
T_{j-1}^{\prime}=T_{j-1} \oplus T_{j-1}^{\prime \prime} \quad \text { and } \quad \iota_{j}^{\prime}(x)=\left(\beta(x), \iota_{j}^{\prime \prime} \alpha^{\prime}(x)\right) \text { for all } \quad x \in W_{j}
$$

we obtain a commutative diagram

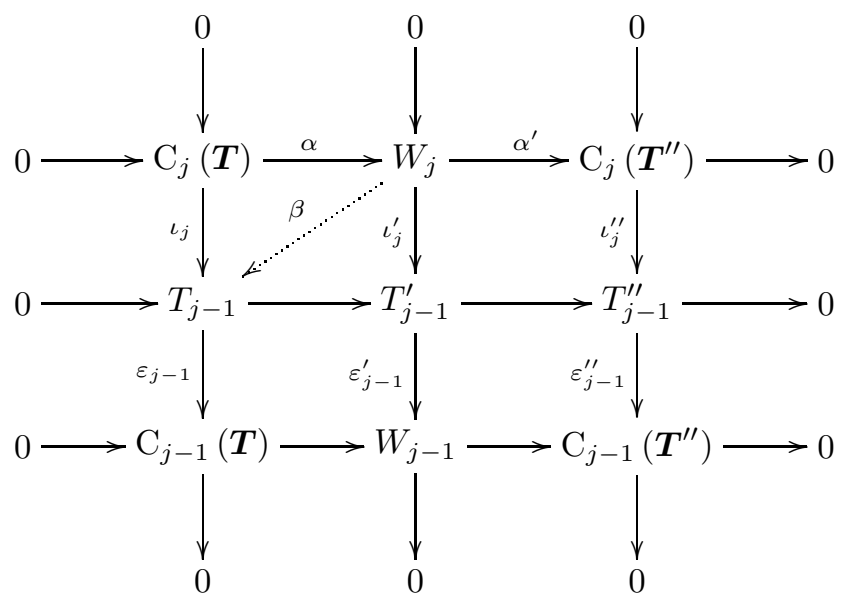

where $W_{j-1}=$ Coker $\iota_{j}^{\prime}$ and $\varepsilon_{j-1}^{\prime}$ is the canonical projection. The columns and the top two rows are exact by construction, hence so is the third row, by the Snake Lemma. To complete the step of the induction, set $\partial_{j}^{\boldsymbol{T}^{\prime}}=\varepsilon_{j}^{\prime} \iota_{j}^{\prime}$.

Step 2. There exists a surjective morphism $\tau^{\prime}$, such that $\tau_{i}^{\prime}=\operatorname{id}_{i}^{T^{\prime}}$ for all $i \geq g$ and the lower part of diagram $(*)$ commutes.

Note first that if $(*)$ commutes, then $\tau^{\prime}$ is surjective because $\tau$ and $\tau^{\prime \prime}$ are.

As the modules $P_{i}$ and $T_{i}^{\prime \prime}$ are projective, we may choose $R$-linear splitting

$$
T_{i}^{\prime}=T_{i} \oplus T_{i}^{\prime \prime} \quad \text { and } \quad P_{i}^{\prime}=P_{i} \oplus P_{i}^{\prime \prime} \quad \text { for all } \quad i \in \mathbb{Z}
$$


such that the maps $\bar{\mu}_{i}$ and $\widehat{\mu}_{i}$ respectively, $\bar{\mu}_{i}^{\prime}$ and $\widehat{\mu}_{i}^{\prime}$, are the canonical injections and respectively, the canonical projections. By [21, (4.6), p. 18] there are then morphisms of complexes

$$
\theta^{\boldsymbol{P}}: \boldsymbol{P}^{\prime \prime} \rightarrow \Sigma \boldsymbol{P} \quad \text { and } \quad \theta^{\boldsymbol{T}}: \boldsymbol{T}^{\prime \prime} \rightarrow \Sigma \boldsymbol{T}
$$

such that the differentials of $\boldsymbol{P}^{\prime}$ and $\boldsymbol{T}^{\prime}$ are given, respectively, by

$$
\partial^{\boldsymbol{P}^{\prime}}=\left(\begin{array}{cc}
\partial^{\boldsymbol{P}} & \theta^{\boldsymbol{P}} \\
0 & \partial^{\boldsymbol{P}^{\prime \prime}}
\end{array}\right) \quad \text { and } \quad \partial^{\boldsymbol{T}^{\prime}}=\left(\begin{array}{cc}
\partial^{\boldsymbol{T}} & \theta^{\boldsymbol{T}} \\
0 & \partial^{\boldsymbol{T}^{\prime \prime}}
\end{array}\right) .
$$

Assume that we have found a homomorphism $\Delta: T^{\prime \prime} \rightarrow P$, satisfying

$$
\Delta_{i-1} \partial_{i}^{\boldsymbol{T}^{\prime \prime}}-\partial_{i}^{\boldsymbol{P}} \Delta_{i}=\theta_{i}^{\boldsymbol{P}} \tau_{i}^{\prime \prime}-\tau_{i-1} \theta_{i}^{\boldsymbol{T}}
$$

for all $i \in \mathbb{Z}$. As in the classical case in [10, (V.2.3)], a simple computation shows that the map $\tau^{\prime}: \boldsymbol{T}^{\prime} \rightarrow \boldsymbol{P}^{\prime}$ defined by

$$
\tau^{\prime}=\left(\begin{array}{cc}
\tau & \Delta \\
0 & \tau^{\prime \prime}
\end{array}\right)
$$

is a morphism making the diagram $(*)$ commutative.

We produce $\Delta_{i}$ by descending induction on $i$. As $\tau_{i}$ and $\tau_{i}^{\prime \prime}$ are identity maps for $i \geq g$, for these $i$ we set $\Delta_{i}=0$. Note that $\tau_{i}^{\prime}=\operatorname{id}^{T_{i}^{\prime}}$ for all $i \geq g$. Let $j$ be an integer such that $j<g$ and assume, by induction, that $\Delta_{j}$ has been constructed and $(1 . j+1)$ holds. We then have

$$
\begin{aligned}
\left(\theta_{j}^{\boldsymbol{P}} \tau_{j}^{\prime \prime}-\tau_{j-1}\right. & \left.\theta_{j}^{\boldsymbol{T}}+\partial_{j}^{\boldsymbol{P}} \Delta_{j}\right) \partial_{j+1}^{\boldsymbol{T}^{\prime \prime}} \\
& =\theta_{j}^{\boldsymbol{P}} \tau_{j}^{\prime \prime} \partial_{j+1}^{\boldsymbol{T}^{\prime \prime}}-\tau_{j-1} \theta_{j}^{\boldsymbol{T}} \partial_{j+1}^{\boldsymbol{T}^{\prime \prime}}+\partial_{j}^{\boldsymbol{P}}\left(\theta_{j+1}^{\boldsymbol{P}} \tau_{j+1}^{\prime \prime}-\tau_{j} \theta_{j+1}^{\boldsymbol{T}}+\partial_{j+1}^{\boldsymbol{P}} \Delta_{j+1}\right) \\
& =\left(\theta_{j}^{\boldsymbol{P}} \tau_{j}^{\prime \prime} \partial_{j+1}^{\boldsymbol{T}^{\prime \prime}}+\partial_{j}^{\boldsymbol{P}} \theta_{j+1}^{\boldsymbol{P}} \tau_{j+1}^{\prime \prime}\right)-\left(\tau_{j-1} \theta_{j}^{\boldsymbol{T}} \partial_{j+1}^{\boldsymbol{T}^{\prime \prime}}+\partial_{j}^{\boldsymbol{P}} \tau_{j} \theta_{j+1}^{\boldsymbol{T}}\right) \\
& =\left(\theta_{j}^{\boldsymbol{P}} \partial_{j+1}^{\boldsymbol{P}^{\prime \prime}}+\partial_{j}^{\boldsymbol{P}} \theta_{j+1}^{\boldsymbol{P}}\right) \tau_{j+1}^{\prime \prime}-\tau_{j-1}\left(\theta_{j}^{\boldsymbol{T}} \partial_{j+1}^{\boldsymbol{T}^{\prime \prime}}+\partial_{j}^{\boldsymbol{T}} \theta_{j+1}^{\boldsymbol{T}}\right) \\
& =0 .
\end{aligned}
$$

Since the complex $\boldsymbol{T}^{\prime \prime}$ is totally acyclic, for $P=P_{j-1}$ we get an exact sequence

$$
\operatorname{Hom}_{R}\left(T_{j-1}^{\prime \prime}, P\right) \stackrel{\operatorname{Hom}_{R}\left(\partial_{j}^{T^{\prime \prime}}, P\right)}{\longrightarrow} \operatorname{Hom}_{R}\left(T_{j}^{\prime \prime}, P\right) \stackrel{\operatorname{Hom}_{R}\left(\partial_{j+1}^{\left.T^{\prime \prime}, P\right)}\right.}{\longrightarrow} \operatorname{Hom}_{R}\left(T_{j+1}^{\prime \prime}, P\right) .
$$

Thus, there exists a homomorphism $\Delta_{j-1}: T_{j-1}^{\prime \prime} \rightarrow P_{j-1}$ satisfying

$$
\Delta_{j-1} \partial_{j}^{\boldsymbol{T}^{\prime \prime}}=\theta_{j}^{\boldsymbol{P}} \tau_{j}^{\prime \prime}-\tau_{j-1} \theta_{j}^{\boldsymbol{T}}+\partial_{j}^{\boldsymbol{P}} \Delta_{j},
$$

that is, formula $(1 . j)$ is satisfied. This finishes the step of the induction.

Now, the proof of the following proposition is parallel to that of [6, (5.6)].

Proposition 4.8. For each exact sequence

$$
M_{\bullet}=0 \rightarrow M \stackrel{\mu}{\rightarrow} M^{\prime} \stackrel{\mu^{\prime}}{\longrightarrow} M^{\prime \prime} \rightarrow 0
$$

of complexes of finite Gorenstein projective dimension and for every complex of $R$-modules $\boldsymbol{N}$ there exist natural in $\boldsymbol{M}_{\bullet}$ and $\boldsymbol{N}$ homomorphisms $\hat{\partial}_{R}^{n}\left(M_{\bullet}, \boldsymbol{N}\right)$, such that the sequence below is exact:

$$
\begin{aligned}
& \cdots \longrightarrow \operatorname{Ext}_{R}^{n}\left(\boldsymbol{M}^{\prime \prime}, \boldsymbol{N}\right) \stackrel{\operatorname{Ext}_{R}^{n}\left(\mu^{\prime}, \boldsymbol{N}\right)}{\longrightarrow} \operatorname{Ext}_{R}^{n}\left(\boldsymbol{M}^{\prime}, \boldsymbol{N}\right) \stackrel{\operatorname{Ext}_{R}^{n}(\mu, \boldsymbol{N})}{\longrightarrow} \operatorname{Ext}_{R}^{n}(\boldsymbol{M}, \boldsymbol{N}) \\
& \stackrel{\hat{\delta}_{R}^{n}\left(M_{\bullet}, \boldsymbol{N}\right)}{\longrightarrow} \mathrm{Ext}_{R}^{n+1}\left(\boldsymbol{M}^{\prime \prime}, \boldsymbol{N}\right) \longrightarrow \quad \cdots
\end{aligned}
$$


and for each $n \in \mathbb{Z}$ there is an equality

$$
\hat{\partial}_{R}^{n}\left(M_{\bullet}, \boldsymbol{N}\right) \circ \hat{\varepsilon}_{R}^{n}(\boldsymbol{M}, \boldsymbol{N})=\hat{\varepsilon}_{R}^{n+1}\left(\boldsymbol{M}^{\prime \prime}, \boldsymbol{N}\right) \circ \varlimsup_{R}^{n}\left(\boldsymbol{M}_{\bullet}, \boldsymbol{N}\right) .
$$

\section{Complexes with Bounded Below homology}

Gorenstein dimension of complexes with bounded below homology admits additional descriptions. In this section we concentrate on such complexes whose Gorenstein projective dimension is finite. First, we note that they form a proper subclass of the class of complexes of finite Gorenstein projective dimension.

Example 5.1. The complex $\boldsymbol{M}=\coprod_{i \leq 0}\left(\Sigma^{i} R\right)$ satisfies

$$
\inf \mathrm{H}(\boldsymbol{M})=-\infty \quad \text { and } \quad \operatorname{Gpd}_{R} \boldsymbol{M}=0 .
$$

Indeed, the first equality is clear. For the second, apply Corollary 3.5 and 3.3 ,

5.2. A Gorenstein projective resolution of $\boldsymbol{M}$ is a complex of Gorenstein projective modules $\boldsymbol{G}$ such that $\boldsymbol{G} \simeq \boldsymbol{M}$. Such a resolution is finite if $G_{i}=0$ for all $|i| \gg 0$; it is special if it is finite, $\inf \boldsymbol{G}=\inf \mathrm{H}(\boldsymbol{M})$, and $G_{i}$ is projective for all $i>\inf \mathrm{H}(\boldsymbol{M})$.

The notion of special Gorenstein projective resolution is an extension to complexes of the notion of finite strict resolution which was defined in the case of finite modules over commutative rings by Avramov and Martsinkovsky in [6]; see also Remark 6.2. It is used in Section [6 to define relative cohomology for arbitrary modules over associative rings.

When given a complex $\boldsymbol{M}$ with bounded below homology, of finite Gorenstein projective dimension, one can easily construct a special Gorenstein projective resolution; see Construction 5.5. The next result, which is proved at the end of this section, shows that in the case of bounded below complexes one can compute the Gorenstein projective dimension using only special resolutions.

Theorem 5.3. If $\boldsymbol{M}$ is a complex with $\inf \mathrm{H}(\boldsymbol{M})>-\infty$, then there are equalities

$$
\begin{aligned}
\operatorname{Gpd}_{R} \boldsymbol{M} & =\inf \left\{\begin{array}{l|l}
\sup \boldsymbol{G} & \begin{array}{c}
\boldsymbol{G} \text { is a finite Gorenstein } \\
\text { projective resolution of } \boldsymbol{M}
\end{array}
\end{array}\right\} \\
& =\inf \left\{\begin{array}{l|l}
\sup \boldsymbol{G} & \begin{array}{l}
\boldsymbol{G} \text { is a special Gorenstein } \\
\text { projective resolution of } \boldsymbol{M}
\end{array}
\end{array}\right\} .
\end{aligned}
$$

In the next remark, we compare our notion of Gorenstein projective dimension for complexes with earlier concepts, which are restricted to special classes of complexes.

Remark 5.4. When $R$ is commutative and noetherian, Yassemi defines in 23] a Gorenstein dimension for complexes $\boldsymbol{M}$ such that $\mathrm{H}(\boldsymbol{M})$ is bounded and $\mathrm{H}_{i}(\boldsymbol{M})$ is finite for all $i \in \mathbb{Z}$. Yassemi says that $\boldsymbol{M}$ has finite Gorenstein dimension if $\mathrm{H}\left(\mathbf{R H o m}_{R}(\boldsymbol{M}, R)\right)$ is finite and the canonical map in the derived category

$$
\boldsymbol{M} \rightarrow \mathbf{R} \operatorname{Hom}_{R}\left(\mathbf{R} \operatorname{Hom}_{R}(\boldsymbol{M}, R), R\right)
$$

is an isomorphism. When this is the case, he calls the number

$$
-\inf \left(\mathrm{H}\left(\mathbf{R} \operatorname{Hom}_{R}(\boldsymbol{M}, R)\right)\right)
$$

the Gorenstein dimension of $R$. When $M$ is a finite $R$-module, he shows in 23, (2.7)] that it is equal to the Gorenstein dimension of Auslander and Bridger [1, $(3.8)]$. 
When $\mathrm{H}(\boldsymbol{M})$ is bounded below, Christensen defines in [12, (4.4.2)] the Gorenstein projection dimension of $\boldsymbol{M}$ to be

$$
\inf \left\{\begin{array}{c|c}
\sup \boldsymbol{G} & \begin{array}{c}
\boldsymbol{G} \text { is a complex of } \\
\text { Gorenstein projective modules with } \\
\inf \boldsymbol{G}>-\infty \text { and } \boldsymbol{G} \simeq \boldsymbol{M}
\end{array}
\end{array}\right\} .
$$

He shows in [12, (2.3.8)] that if the hypotheses of Yassemi's definition are satisfied, then the two Gorenstein dimensions coincide.

The first equality in Theorem 5.3 shows that for bounded below complexes our definition of Gorenstein projective dimension coincides with that of Christensen.

For the rest of this section, unless otherwise specified, $\boldsymbol{M}$ is a complex with

$$
\inf \mathrm{H}(\boldsymbol{M})=t<\infty .
$$

Construction 5.5. Suppose $\operatorname{Gpd}_{R} \boldsymbol{M}=g<\infty$. By 1.3.4 and Theorem 3.4 choose a surjective complete resolution $\boldsymbol{T} \stackrel{\tau}{\rightarrow} \boldsymbol{P} \stackrel{\pi}{\rightarrow} \boldsymbol{M}$ with $\inf \boldsymbol{P}=t$ and $\tau_{i}$ bijective for all $i \geq g$. Set $\boldsymbol{L}=\operatorname{Ker} \tau$ and form a complex $\left(\boldsymbol{G}, \partial^{\boldsymbol{G}}\right)$ by setting

$$
G_{i}=\left\{\begin{array}{ll}
0 & \text { for } i<t ; \\
\mathrm{C}_{t}(\boldsymbol{T}) & \text { for } i=t ; \\
L_{i-1} & \text { for } i>t ;
\end{array} \quad \text { and } \quad \partial_{i}^{\boldsymbol{G}}= \begin{cases}0 & \text { for } i<t+1 ; \\
-\varsigma & \text { for } i=t+1 ; \\
-\partial_{i-1}^{L} & \text { for } i>t+1,\end{cases}\right.
$$

where $\varsigma: L_{t} \rightarrow \mathrm{C}_{t}(\boldsymbol{T})$ is the natural homomorphism of modules.

Let $\iota: \boldsymbol{G} \rightarrow \Sigma \boldsymbol{L}$ be the morphism of complexes given by

$$
\iota_{i}= \begin{cases}0 & \text { for } i<t \\ \varsigma^{\prime} & \text { for } i=t \\ \mathrm{id}^{L_{i-1}} & \text { for } i>t\end{cases}
$$

where $\varsigma^{\prime}: \mathrm{C}_{t}(\boldsymbol{T}) \rightarrow T_{t-1}=L_{t-1}$ is the canonical inclusion.

Let $\lambda: \boldsymbol{P} \rightarrow \Sigma \boldsymbol{L}$ be the morphism defined in 2.2 .3 via 1.2 .3 and define a map $\gamma: \boldsymbol{P} \rightarrow \boldsymbol{G}$ by the formulas

$$
\gamma_{i}= \begin{cases}0 & \text { for } i<t \\ \varsigma^{\prime \prime} & \text { for } i=t \\ \lambda_{i} & \text { for } i>t\end{cases}
$$

where $\varsigma^{\prime \prime}: P_{t} \rightarrow \mathrm{C}_{t}(\boldsymbol{T})$ is the natural homomorphism of modules induced by $\lambda_{t}$.

Lemma 5.6. With the notation above, the diagram

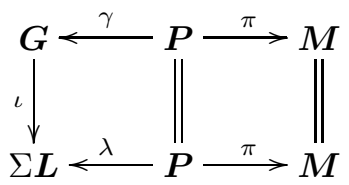

is commutative and all arrows are quasi-isomorphisms. In particular, $\boldsymbol{G}$ is a special Gorenstein projective resolution of $\boldsymbol{M}$ with $\sup \boldsymbol{G}=g$.

When $\boldsymbol{M}$ is a module, $M$, there is a quasi-isomorphism $\varepsilon: \boldsymbol{G} \rightarrow M$ with $\varepsilon \gamma=\pi$. 
Proof. Commutativity follows from the definitions of the maps. The definition of $\boldsymbol{G}$, the equality $L_{\leq t-1}=T_{\leq t-1}$ and the exactness of $\boldsymbol{T}$ yield

$$
\mathrm{H}_{i}(\boldsymbol{G})= \begin{cases}0 & \text { for } i<t \\ \mathrm{H}_{i-1}(\boldsymbol{L}) & \text { for } i \geq t\end{cases}
$$

Therefore, $\iota$ is a quasi-isomorphism. The morphism $\lambda$ is a quasi-isomorphism by 2.2.3. hence from the equality $\iota \gamma=\lambda$ we obtain that $\gamma$ is a quasi-iomorphism. We have $\sup \boldsymbol{G}=g$ by Construction 5.5 .

If $\boldsymbol{M}=M$ is a module, then $t=0, \boldsymbol{P}$ becomes a projective resolution of $M=\mathrm{C}_{0}(\boldsymbol{P})$ and $\tau_{0}: T_{0} \rightarrow P_{0}$ induces a map $\bar{\tau}_{0}: \mathrm{C}_{0}(\boldsymbol{T}) \rightarrow \mathrm{C}_{0}(\boldsymbol{P})$. Setting $\varepsilon_{i}=0$ for $i \neq 0$ and $\varepsilon_{0}=\bar{\tau}_{0}$ we get a quasi-isomorphism $\varepsilon: \boldsymbol{G} \rightarrow M$ with $\varepsilon \gamma=\pi$.

Construction 5.7. Suppose that $G \simeq M$ is a special Gorenstein projective resolution. By definition, the module $G_{t}$ is Gorenstein projective, so there exists a totally acyclic complex $\boldsymbol{S}$ with $\mathrm{C}_{t+1}(\boldsymbol{S})=G_{t}$.

Let $\left(\boldsymbol{K}, \partial^{\boldsymbol{K}}\right)$ denote the complex of projectives with

$$
K_{i}=\left\{\begin{array}{ll}
S_{i} & \text { for } i \leq t ; \\
G_{i} & \text { for } i>t ;
\end{array} \quad \text { and } \quad \partial_{i}^{\boldsymbol{K}}= \begin{cases}\partial_{i}^{\boldsymbol{S}} & \text { for } i<t+1 ; \\
\varepsilon \circ \partial_{t+1}^{\boldsymbol{G}} & \text { for } i=t+1 ; \\
\partial_{i}^{\boldsymbol{G}} & \text { for } i>t+1,\end{cases}\right.
$$

where $\varepsilon: G_{t} \rightarrow S_{t}$ is the canonical injection.

Let $\iota^{\prime}: \boldsymbol{G} \rightarrow \boldsymbol{K}$ be the morphism of complexes given by

$$
\iota_{i}^{\prime}= \begin{cases}0 & \text { for } i<t \\ \varepsilon & \text { for } i=t \\ \mathrm{id}^{G_{i}} & \text { for } i>t .\end{cases}
$$

Lemma 5.8. With the notation above, for any semiprojective resolution $\boldsymbol{P} \stackrel{\pi}{\rightarrow} \boldsymbol{M}$, there exists a commutative diagram of quasi-isomorphisms:

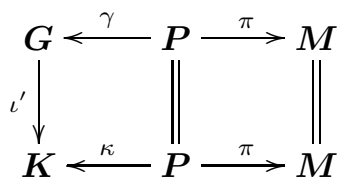

If $\inf \boldsymbol{P}=t<\infty$, then there exists a surjective complete resolution

$$
\boldsymbol{T} \stackrel{\tau}{\rightarrow} \boldsymbol{P} \stackrel{\pi}{\rightarrow} M
$$

with $\operatorname{Ker} \tau=\Sigma^{-1} \boldsymbol{K}$.

Proof. A quasi-isomorphism $\gamma$ is obtained from 1.3.2. It is easy to see that $\iota^{\prime}$ is a quasi-isomorphism. To complete the square on the right-hand side, set $\kappa=\iota^{\prime} \gamma$.

Assume inf $\boldsymbol{P}=t<\infty$. Set $\boldsymbol{T}=\Sigma^{-1} \operatorname{Cone}(\kappa)$; this is a complex of projectives, and it is exact because $\kappa$ is a quasi-isomorphism. For all $i \leq t$ we have $\mathrm{C}_{i}(\boldsymbol{T})=$ $\mathrm{C}_{i}(\boldsymbol{S})$ by construction, so the module $\mathrm{C}_{i}(\boldsymbol{T})$ is Gorenstein projective; Lemma 2.3 .3 now shows that $\boldsymbol{T}$ is totally acyclic. The exact sequence of complexes

$$
0 \rightarrow \Sigma^{-1} \boldsymbol{K} \rightarrow \boldsymbol{T} \stackrel{\tau}{\rightarrow} \boldsymbol{P} \rightarrow 0
$$

gives a surjective complete resolution with the desired property. 
Remark. The reader might note that Constructions 5.5] and 5.7 are "inverse to each other".

Proof of Theorem [5.3. If $\inf \mathrm{H}(\boldsymbol{M})=\infty$, then $\boldsymbol{M}$ is exact and $\mathbf{0} \cong \boldsymbol{M}$ is a finite (special) Gorenstein projective resolution, therefore the infima of the sets in the right-hand side are $-\infty$. By $3.2, \operatorname{Gpd}_{R} M=-\infty$, so the theorem holds in this case. Thus, we may assume that $\inf \mathrm{H}(\boldsymbol{M})$ is finite.

Set $\operatorname{Gpd}_{R} \boldsymbol{M}=g$ and let $h$ (respectively, $h^{\prime}$ ) denote the number on the righthand side of the first (respectively, second) equality of the theorem.

We show first $g \leq h$. If $h=\infty$, it is clear. If $h<\infty$, then there exists a finite Gorenstein projective resolution $\boldsymbol{G}$ of $\boldsymbol{M}$; set $s=\sup \boldsymbol{G}$. Let $\boldsymbol{P} \rightarrow \boldsymbol{M}$ be a semiprojective resolution with $P_{i}=0$ for all $i<t$ and let $\gamma: \boldsymbol{P} \rightarrow \boldsymbol{G}$ be a quasi-isomorphism given by 1.3.1 The mapping cone $\operatorname{Cone}(\gamma)$ is an exact complex of Gorenstein projective modules, bounded below, so every syzygy is a Gorenstein projective module by 2.3.5, in particular, so is $\mathrm{C}_{s+1}(\operatorname{Cone}(\gamma))=\mathrm{C}_{s}(\boldsymbol{P})$. As $\boldsymbol{G}$ is quasi-isomorphic to $\boldsymbol{M}$, sup $\mathrm{H}(\boldsymbol{M}) \leq s$. From Theorem 3.4 we get $g \leq s$, so $g \leq h$.

The inequality $h \leq h^{\prime}$ is clear.

We show next $h^{\prime} \leq g$. If $g=\infty$, it is clear. If $g<\infty$, then by Lemma 5.6 there exists a special Gorenstein projective resolution $\boldsymbol{G}$ of $\boldsymbol{M}$ such that $\sup \boldsymbol{G}=g$.

\section{Relative COHOMOLOGY FOR MOdUles}

In this section $R$ is an associative ring and $M$ is a left $R$-module.

When $R$ is two-sided noetherian and $M$ admits a "proper" resolution by finite modules of Gorenstein dimension zero, Avramov and Martinkovsky [6 associate to $M$ relative cohomology groups. They study the behavior of these groups with respect to short exact sequences, and relate them to the absolute cohomology groups and to the Tate cohomology groups.

Holm [19] extends the definition of relative cohomology groups to the case when $M$ admits a proper resolution by Gorenstein projective modules over an arbitrary ring $R$. He focuses on the relation of these relative groups with those defined using a proper resolution of the second argument by Gorenstein injective modules.

Our purpose here is to show that the result of [6] extends to the general setup of 19. As most arguments carry over, we just give indications of proofs. In Remark 6.7 we comment on obstacles to defining relative cohomology groups for complexes.

6.1. A complex $\boldsymbol{C}$ is called proper exact if $\operatorname{Hom}_{R}(E, C)$ is exact for all Gorenstein projective modules E. A proper Gorenstein projective resolution of $M$ is a complex of Gorenstein projective modules $\boldsymbol{G}$ together with a morphism $\varepsilon: \boldsymbol{G} \rightarrow M$, such that the complex Cone $(\varepsilon)$ is proper exact.

Let $\mathcal{G P}$ denote the class of Gorenstein projective modules and let $\overline{\mathcal{G P}}$ denote the class of modules that admit some proper resolution.

Remark 6.2. Let $M$ be an $R$-module with $\operatorname{Gpd}_{R} M<\infty$. By Lemma 5.6 there exists a special Gorenstein projective resolution $\boldsymbol{G} \stackrel{\gamma}{\leftarrow} \boldsymbol{P} \stackrel{\pi}{\longrightarrow} M$ and a quasiisomorphism $\varepsilon: G \rightarrow M$ such that $\varepsilon \gamma=\pi$. Such a resolution is called finite strict in [6] and any finite strict resolution is proper by [6, (4.1.3)]. Thus, every module of finite Gorenstein projective dimension is in $\overline{\mathcal{G P}}$. 
6.3. For each $M \in \overline{\mathcal{G P}}$ choose a proper resolution $\boldsymbol{G} \rightarrow M$. For each $R$-module $N$ and each $n \in \mathbb{Z}$, the $n^{\text {th }}$ relative cohomology group is defined by

$$
\operatorname{Ext}_{\mathcal{G P}}^{n}(M, N)=\mathrm{H}^{n} \operatorname{Hom}_{R}(\boldsymbol{G}, N) .
$$

Choose a projective resolution $\boldsymbol{P} \rightarrow M$ and a morphism $\gamma: \boldsymbol{P} \rightarrow \boldsymbol{G}$ lifting the identity on $M$. The morphism of complexes of abelian groups

$$
\operatorname{Hom}_{R}(\gamma, N): \operatorname{Hom}_{R}(\boldsymbol{G}, N) \rightarrow \operatorname{Hom}_{R}(\boldsymbol{P}, N)
$$

induces for every $n \in \mathbb{Z}$ a natural homomorphism of abelian groups

$$
\varepsilon_{\mathcal{G P}}^{n}(M, N): \operatorname{Ext}_{\mathcal{G P}}^{n}(M, N) \rightarrow \operatorname{Ext}_{R}^{n}(M, N) .
$$

The groups and maps defined above do not depend on the choices of resolutions and liftings used in their constructions; see [6, (4.2)] or [19, (2.4)].

The following two propositions correspond to [6, (4.6)], 6, (4.4)], whose proofs extend verbatim to the more general setting below.

Proposition 6.4. For each proper exact sequence $\boldsymbol{M}=0 \rightarrow M \stackrel{\mu}{\rightarrow} M^{\prime} \stackrel{\mu^{\prime}}{\rightarrow} M^{\prime \prime} \rightarrow 0$ of $R$-modules in $\overline{\mathcal{G P}}$ and each $R$-module $N$ there are natural in $\boldsymbol{M}$ and $N$ homomorphisms $\widetilde{\partial}_{\mathcal{G} \mathcal{P}}^{n}(\boldsymbol{M}, N)$, such that the sequence below is exact:

$$
\begin{aligned}
& \cdots \longrightarrow \operatorname{Ext}_{\mathcal{G P}}^{n}\left(M^{\prime \prime}, N\right) \stackrel{\operatorname{Ext}_{\mathcal{G P}}^{n}\left(\mu^{\prime}, N\right)}{\longrightarrow} \operatorname{Ext}_{\mathcal{G P}}^{n}\left(M^{\prime}, N\right) \stackrel{\operatorname{Ext}_{\mathcal{G P}}^{n}(\mu, N)}{\longrightarrow} \operatorname{Ext}_{\mathcal{G P}}^{n}(M, N) \\
& \stackrel{\partial_{\mathcal{G P}}^{n}(M, N)}{\longrightarrow} \operatorname{Ext}_{\mathcal{G P}}^{n+1}\left(M^{\prime \prime}, N\right) \longrightarrow \quad \cdots
\end{aligned}
$$

and for each $n \in \mathbb{Z}$ there is an equality

$$
\varlimsup_{R}^{n}(M, N) \circ \varepsilon_{\mathcal{G P}}^{n}(M, N)=\varepsilon_{\mathcal{G P}}^{n+1}\left(M^{\prime \prime}, N\right) \circ \varlimsup_{\mathcal{G} \mathcal{P}}^{n}(M, N) .
$$

Proposition 6.5. For each $R$-module $M \in \overline{\mathcal{G P}}$ and each proper exact sequence $\boldsymbol{N}=0 \rightarrow N \stackrel{\nu}{\rightarrow} N^{\prime} \stackrel{\nu^{\prime}}{\rightarrow} N^{\prime \prime} \rightarrow 0$ of $R$-modules there exist natural in $M$ and $\boldsymbol{N}$ homomorphisms $\mathrm{\partial}_{\mathcal{G} \mathcal{P}}^{n}(M, N)$, such that the sequence below is exact:

$$
\begin{gathered}
\cdots \longrightarrow \stackrel{\longrightarrow}{\longrightarrow} \operatorname{Ext}_{\mathcal{G P}}^{n}(M, N) \stackrel{\operatorname{Ext}_{\mathcal{G P}}^{n}(M, \nu)}{\longrightarrow} \operatorname{Ext}_{\mathcal{G} \mathcal{P}}^{n}\left(M, N^{\prime}\right) \stackrel{\operatorname{Ext}_{\mathcal{G P}}^{n}\left(M, \nu^{\prime}\right)}{\longrightarrow} \operatorname{Ext}_{\mathcal{G P}}^{n}\left(M, N^{\prime \prime}\right) \\
\stackrel{\delta_{\mathcal{G} \mathcal{P}}^{n}(M, N)}{\longrightarrow} \operatorname{Ext}_{\mathcal{G P}}^{n+1}(M, N) \longrightarrow
\end{gathered}
$$

and for each $n \in \mathbb{Z}$ there is an equality

$$
\widetilde{\partial}_{R}^{n}(M, N) \circ \varepsilon_{\mathcal{G P}}^{n}\left(M, N^{\prime \prime}\right)=\varepsilon_{\mathcal{G P}}^{n+1}(M, N) \circ \varlimsup_{\mathcal{G} \mathcal{P}}^{n}(M, N) .
$$

When $\operatorname{Gpd}_{R} M=g<\infty$, Remark 4.2 describes the map $\hat{\varepsilon}_{R}(M, N)$ for $n<0$ and $n>g$. The next result analyzes this map for $1 \leq n \leq g$. It extends 6 , (7.1)], whose proof also carries over. We indicate how the original argument can be shortened.

Theorem 6.6. Let $M$ be a module with $\operatorname{Gpd}_{R} M=g<\infty$. For each $R$-module $N$ there exist homomorphisms $\hat{\delta}_{R}^{n}(M, N)$, natural in $M$ and $N$, such that the following 
sequence is exact:

$$
\begin{aligned}
0 \longrightarrow & \operatorname{Ext}_{\mathcal{G P}}^{1}(M, N) \stackrel{\varepsilon_{\mathcal{G P}}^{1}(M, N)}{\longrightarrow} \operatorname{Ext}_{R}^{1}(M, N) \stackrel{\cdots}{\longrightarrow} \operatorname{Ext}_{\mathcal{G P}}^{n}(M, N) \stackrel{\varepsilon_{\mathcal{P} \mathcal{P}}^{n}(M, N)}{\longrightarrow} \operatorname{Ext}_{R}^{n}(M, N) \stackrel{\hat{\varepsilon}_{R}^{n}(M, N)}{\longrightarrow} \operatorname{Ext}_{R}^{n}(M, N) \\
\longrightarrow & \longrightarrow \operatorname{Ext}_{R}^{g}(M, N) \longrightarrow
\end{aligned}
$$

Proof. As in the proof of [6, (7.1)], but using Lemma 5.8 instead of [6, (3.8)], we obtain a split exact sequence

$$
0 \rightarrow \operatorname{Hom}_{R}(\boldsymbol{P}, N) \rightarrow \operatorname{Hom}_{R}\left(\boldsymbol{T}^{b}, N\right) \rightarrow \operatorname{Hom}_{R}\left(\Sigma^{-1} \boldsymbol{G}, N\right) \rightarrow 0 .
$$

Its long cohomology sequence

$$
\begin{aligned}
& \cdots \stackrel{\hat{\delta}_{R}^{n-1}(M, N)}{\longrightarrow} \operatorname{Ext}_{\mathcal{G P}}^{n}(M, N) \stackrel{\partial^{n-1}}{\longrightarrow} \operatorname{Ext}_{R}^{n}(M, N) \\
& \stackrel{\mathrm{H}^{n}\left(\operatorname{Hom}_{R}\left(\tau^{\mathrm{b}}, N\right)\right)}{\longrightarrow} \mathrm{H}^{n}\left(\operatorname{Hom}_{R}\left(\boldsymbol{T}^{\mathrm{b}}, N\right)\right)
\end{aligned}
$$

induces the desired long exact sequence of the theorem, which is natural in $M$ and $N$.

Here we remark that the naturality of the map $\partial^{n-1}$, follows by general theory. Indeed, the exact sequence $(*)$ is obtained as follows: we consider the cone exact sequence of the map $\gamma: \boldsymbol{P} \rightarrow \boldsymbol{G}$ of Lemma 5.8 and we apply $\Sigma^{-1}$ to it, so we obtain the split exact sequence

$$
0 \rightarrow \Sigma^{-1} \boldsymbol{G} \rightarrow \boldsymbol{T}^{b} \rightarrow \boldsymbol{P} \rightarrow 0 .
$$

Applying $\operatorname{Hom}_{R}(-, N)$, we get $(*)$. By 1.2 .3 and 1.2 .2 we obtain the expression

$$
\check{\partial}^{n-1}=(-1)^{n} \varepsilon_{\mathcal{G P}}^{n}(M, N) \text { for all } n>0
$$

for the connecting homomorphism $ð$.

Remark 6.7. Let $\boldsymbol{M}$ be a complex with $\operatorname{Gpd}_{R} \boldsymbol{M}<\infty$ and inf $\mathrm{H}(\boldsymbol{M})>\infty$. By Lemma [5.6] choose a special Gorenstein projective resolution $\boldsymbol{G} \simeq \boldsymbol{M}$. If $\boldsymbol{M}=$ $M$ and $N$ are modules, then $\mathrm{H}^{n}\left(\operatorname{Hom}_{R}(\boldsymbol{G}, N)\right)$ is the relative cohomology group $\operatorname{Ext}_{\mathcal{G P}}^{n+1}(M, N) ;$ see 6.3

It is tempting to use $\mathrm{H}^{n}\left(\operatorname{Hom}_{R}(\boldsymbol{G}, \boldsymbol{N})\right)$ to define relative cohomology groups for complexes. However, I do not know whether this construction has the necessary uniqueness and functoriality. More precisely, if $\boldsymbol{M}^{\prime}$ is a complex with $\operatorname{Gpd}_{R} \boldsymbol{M}^{\prime}<$ $\infty$ and $\inf \mathrm{H}(\boldsymbol{M}) \geq \inf \mathrm{H}\left(\boldsymbol{M}^{\prime}\right)>\infty$, then for each morphism $\mu: \boldsymbol{M} \rightarrow \boldsymbol{M}^{\prime}$ there exists a morphism of complexes $\widehat{\mu}: \boldsymbol{G} \rightarrow \boldsymbol{G}^{\prime}$ such that if $\mu$ is an isomorphism (in particular, if $\left.\mu=\mathrm{id}^{M}\right)$, then so is $\mathrm{H}^{n}\left(\operatorname{Hom}_{R}(\widehat{\mu}, \boldsymbol{N})\right)$. I do not know whether this map depends on the choice of $\widehat{\mu}$.

\section{ACKNOWLEDGMEnTs}

I would like to thank my thesis adviser Luchezar Avramov for his patient guidance throughout the development and writing of this article. I thank Sean SatherWagstaff for pointing me to Iversen's book 21, Anders Frankild and Henrik Holm for comments on an earlier version, and Srikanth Iyengar for Theorem 3.11. I thank the referee for a careful reading of the paper and for many useful suggestions. 


\section{REFERENCES}

[1] Auslander, M., Bridger, M., Stable module theory, Mem. Amer. Math. Soc. 94 (1969). MR0269685 (42:4580)

[2] Avramov, L.L., Homological dimensions and related invariants of modules over local rings, Representations of Algebras, ICRA IX (Beijing, 2000), vol. I, Beijing Normal Univ. Press 2002, 1-39. MR2067368

[3] Avramov, L.L., Foxby, H.-B., Homological dimension of unbounded complexes, J. Pure Appl. Alg. 71 (1991), 129-155. MR1117631 (93g:18017)

[4] Avramov, L.L., Foxby, H.-B., Halperin, S., Differential graded homological algebra, Preprint, version of $09 / 11 / 2004$.

[5] Avramov, L.L., Gasharov, V.N., Peeva, I.V., Complete intersection dimension, Publ. Math. I.H.E.S., 86 (1997), 67-114. MR1608565 (99c:13033)

[6] Avramov, L.L., Martsinkovsky, A., Absolute, relative, and Tate cohomology of modules of finite Gorenstein dimension, Proc. London Math. Soc. (3) 85 (2002), 393-440. MR1912056 (2003g:16009)

[7] Bourbaki, N., Algèbre Chaptre 10, Algèbre Homologique, Masson, Paris, New York, 1980. MR.0610795 (82j:18022)

[8] Bruns, W., Herzog, J., Cohen Macaylay ring, (revised edition), Advances in Mathematics, Vol. 39, Cambridge Univ. Press, Cambridge, UK, 1996. MR.1251956 (95h:13020)

[9] Buchweitz, R.-O., Maximal Cohen-Macaulay modules and Tate cohomology over Gorenstein rings, Preprint, Univ. Hannover, 1986.

[10] Cartan, H., Eilenberg, S., Homological Algebra, Princeton University Press, Princeton, NJ, reprint 1999. MR.1731415(2000h:18022)

[11] Cornick, J., Kropholler, P.H., On complete resolutions, Topology Appl. 78 (1997), 235-250. MR1454602 (98k:20087)

[12] Christensen, L.W., Gorenstein dimensions, Lecture Notes in Math. 1747, Springer, Berlin, 2000. MR1799866 (2002e:13032)

[13] Enochs, E.E., Jenda, O.M.G., Gorenstein injective and projective modules, Math. Z. 220, (1995), 611-633. MR.1363858 (97c:16011)

[14] Enochs, E.E., Jenda, O.M.G., Relative Homological Algebra, De Gruyter Exp. Math. 30, De Gruyter, Berlin, 2000. MR1753146 (2001h:16013)

[15] Farrell, F.T., An extension of Tate cohomology to a class of infinite groups, J. Pure Appl. Alg. 10 (1977), 153-161. MR0470103 (57:9870)

[16] Gedrich, T.V., Gruenberg, K.W., Complete cohomological functors on groups, Topology Appl. 25 (1987), 203-223. MR0884544 (89h:20073)

[17] Gerko, A.A., On homological dimensions, Sb. Math. 192 (2001), 1165-1179. MR1862245 (2002h:13024)

[18] Goto, S., $V$ anishing of $\operatorname{Ext}_{A}^{i}(M, A)$, J. Math. Kyoto Univ. 22 (1982), 481-484. MR0674605 (84c:13019)

[19] Holm, H., Gorenstein derived functors, Proc. Amer. Math. Soc., 132 (2004), 1913-1923. MR2053961

[20] Holm, H., Gorenstein homological dimensions, J. Pure Appl. Alg. 189 (2004), 167-193. MR2038564 (2004k:16013)

[21] Iversen, B., Cohomology of Sheaves, Springer-Verlag, Berlin-Heidelberg, 1986. MR0842190 (87m:14013)

[22] Sather-Wagstaff, S., Complete intersection dimension for complexes, J. Pure Appl. Alg. 190 (2004), 267-290. MR2043332

[23] Yassemi, S., Gorenstein dimension, Math. Scand. 77 (1995), 161-174. MR1379262 (97d:13017)

[24] Veliche, O., Construction of modules of finite Gorenstein dimension, J. Algebra 250 (2002), 427-449. MR.1899298 (2003e:13023)

[25] Weibel, C.,A., An introduction to homological algebra, Cambridge Studies in advanced mathematics 38, Cambridge University Press, Cambridge, 1994. MR.1269324 (95f:18001)

Department of Mathematics, Purdue University, West Lafayette, Indiana 47907

E-mail address: oveliche@math.purdue.edu

E-mail address: oveliche@math.utah.edu 